\title{
Multi-Source Backlogged Probabilistic Inventory Model for Crisp and Fuzzy Environment
}

\author{
H. A. Fergany'a, O. M. Hollah ${ }^{b}$

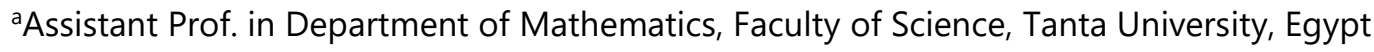 \\ ${ }^{\text {b} A s s i s t a n t ~ L e c t u r e r ~ i n ~ D e p a r t m e n t ~ o f ~ M a t h e m a t i c s, ~ H i g h e r ~ I n s t i t u t e ~ f o r ~ C o m p u t e r s, ~ I n f o r m a t i o n ~ \& ~}$ \\ Management, Tanta, Egypt \\ halafergany@Yahoo.com \& hala.fergany@science.tanta.edu.eg, eng_Hollah@Yahoo.com \& \\ osama.hollah@science.tanta.edu.eg,
}

\begin{abstract}
This paper proposed a multi-item multi-source probabilistic periodic review inventory model under a varying holding cost constraint with zero lead time when: (1) the stock level decreases at a uniform rate over the cycle. (2) some costs are varying. (3) the demand is a random variable that follows some continuous distributions as (two-parameter exponential, Kumerswamy, Gamma, Beta, Rayleigh, Erlang distributions).

The objective function under a constraint is imposed here in crisp and fuzzy environment. The objective is to find the optimal maximum inventory level for a given review time that minimize the expected annual total cost. Furthermore, a comparison between given distributions is made to find the optimal distribution that achieves the model under considerations. Finally, a numerical example is applied.
\end{abstract}

Keywords: Constrained model; Continuous distributions; Lagrange multiplier technique; Periodic inventory model; Varying costs.

\section{Introduction}

In the practical situation, some costs are relating to some variables such as quantity $(Q)$ or length of the cycle $(N)$... etc.,. Many researchers dealing with inventory models with varying costs for example, Chu et al. [6] and Fergany [8] illustrated probabilistic multi-item inventory model with varying mixture shortage cost under restrictions. Fergany and El-Wakeel [10] illustrated probabilistic single item inventory problem with varying order cost under two linear constraints. Abuo - El-Ata et al. [1] introduced probabilistic multi-item inventory model with varying order cost under two restrictions using a geometric programming approach. And other researchers concern with periodic review model for example, Silver and Robb [14] presented the model with some insights regarding the optimal reorder period. Fergany et al. [9] illustrated the model using Lagrange multiplier technique and fuzzy adaptive particle swarm optimization. Chiang [5] illustrated the model with stochastic supplier's visit intervals. Chiang [4] developed optimal replenishment for the model with two supply modes. Chiang [3] presented optimal ordering policies for the model with a refined intra-cycle time scale. Chiang [2] developed the model with a refined delivery scenario. Konstantaras and Papachristos [11] introduced manufacturing and logistics optimal policy and holding cost stability regions in the model with manufacturing and remanufacturing options. Yuyue and Hoong [15] developed the model with application to the continuous review obsolescence problem.

The cost parameters in real inventory systems and other parameters such as price, marketing and service elasticity to demand are imprecise and uncertain in nature. Since the proposed model is in a fuzzy environment, a fuzzy decision should be made to meet the decision criteria, and the results should be fuzzy as well. Fuzzy sets introduced by many researchers as a mathematical way of representing impreciseness or vagueness in everyday life. Rong et al. [12] presented a multi-objective wholesaler-retailers inventory-distribution model with controllable lead-time based on probabilistic fuzzy set and triangular fuzzy number. Sadjadi et al. [13] introduced fuzzy pricing and marketing planning model using a geometric programming approach. 
This paper is formulated a multi-item multi-source periodic review inventory problem with a varying holding cost constraint when the holding and backlogged costs are varying. Also, shortages are permitted but fully backlogged and the demand considered to be a random variable that follows some continuous distributions as (two-parameter exponential, Kumerswamy, Gamma, Beta, Erlang, Raylieph distributions) without lead time. Also, the cost parameters under a constraint is considered here in crisp and fuzzy environment. The problem has been solved by Lagrange multiplier technique. The objective is to find the optimal maximum inventory level for a given review time which minimize the expected annual total cost under a restriction. And a comparison between given distributions is made to find the optimal distribution that achieves the model under considerations The results of the numerical example are got by Mathematica program.

\section{Notations}

Parameters for the $r^{\text {th }}(r=1,2, \ldots, n)$ item, $s^{\text {th }}(s=1,2, \ldots, m)$ source are:

$Q_{m r s} \quad$ the maximum inventory level for $r^{\text {th }}$ item, $s^{\text {th }}$ source (decision variable).

$N \quad$ the time of review (the cycle).

$x_{r s} \quad$ the demand of the $r^{\text {th }}$ item, $s^{\text {th }}$ source during the cycle $N$ (random variable).

$C_{\text {ors }} \quad$ the order cost per unit item for $r^{\text {th }}$ item, $s^{\text {th }}$ source

$C_{\text {prs }} \quad$ the purchase cost for $r^{\text {th }}$ item for $r^{\text {th }}$ item, $s^{\text {th }}$ source

$C_{h r} \quad$ the holding cost per unit item for $r^{\text {th }}$ item.

$C_{b r s} \quad$ the backlogged cost per unit item for $r^{\text {th }}$ item, $s^{\text {th }}$ source

$C_{h r}(N) \quad$ the varying holding cost for the $r^{\text {th }}$ per cycle $=C_{h r} N^{-\beta_{r}}$

$C_{b r s}(N) \quad$ the varying backlogged cost for the $r^{t h}$ per cycle $=C_{b r} N^{\beta_{r}}$

$\bar{D}_{r} \quad$ the expected demand per unit item $\bar{D}_{r} \equiv\left(\bar{D}_{1}, \bar{D}_{2}, \ldots, \bar{D}_{n}\right)$

$k_{\text {hrs }} \quad$ the goal associated to expected holding cost

$\tilde{C}_{\text {ors }} \quad$ the fuzzy set up cost per unit item per unit time.

$\tilde{C}_{h r} \quad$ the fuzzy holding cost per unit item per unit time.

$\tilde{C}_{b r s} \quad$ the fuzzy backlogged cost per unit item per unit time.

$\tilde{C}_{h r}(N) \quad$ the fuzzy varying holding cost for the $r^{t h}$ per cycle $=\tilde{C}_{h r} N^{-\tilde{\beta}_{r}}$

$\tilde{C}_{b r s}(N) \quad$ the fuzzy varying backlogged cost for the $r^{t h}$ per cycle $=\tilde{C}_{b r} N^{\widetilde{\beta}_{r}}$

$\tilde{k}_{h r s} \quad$ the fuzzy goal associated to expected holding cost

$E(T C) \quad$ the expected total average cost function $E\left(T C\left(Q_{m r s}, N\right)\right)$

\section{The mathematical model}

This model developed the stock level decreases at a uniform rate over the cycle. Figure 2 exhibits the inventory flow process two final conditions may arise as indicated in Figure 1. 


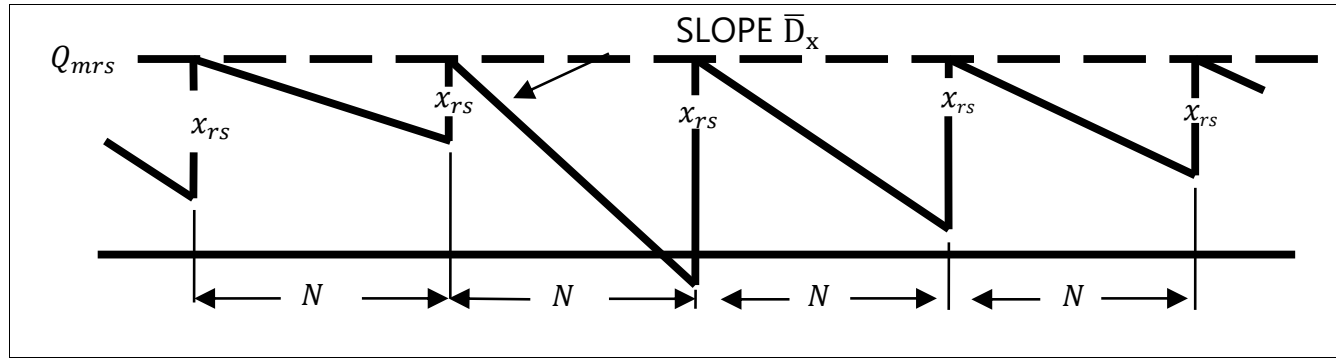

Figure (1): Inventory process with uniform demand

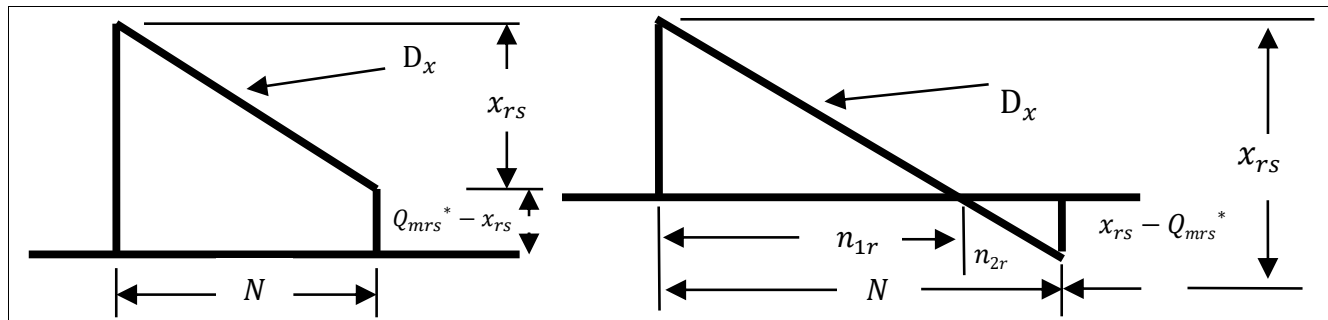

First situation

second situation

Figure (2): Two states of uniform model

The expected total cost of the cycle for multi-item multi source will be the sum of the expected purchase cost, the expected order cost, the expected varying holding cost, and the expected varying backlogged cost,

$$
E(T C)=\sum_{r=1}^{n}\left[E\left(P C_{r s}\right)+E\left(O C_{r s}\right)+E\left(H C_{r s}(N)\right)+E\left(B C_{r s}(N)\right)\right]
$$

The expected purchase cost for the cycle is given by

$E\left(P C_{r s}\right)=C_{p r s} \bar{x}_{r s}$

The expected order cost for the cycle is given by

$E\left(O C_{r s}\right)=C_{\text {ors }}$

The expected varying holding cost for the cycle is given by

$E\left(H C_{r s}\right)=C_{h r}(N) \bar{I}=C_{h r} N^{-\beta_{r}} \bar{I}$

where $\bar{I}$ represents the expected average amount of inventory.

The first situation in Figure (2). If $\bar{x}_{r s} \leq Q_{m r}$. Then the average amount in inventory $\bar{I}$, is given by

$\bar{I}=\frac{Q_{m r s}+\left(Q_{m r s}-x_{r s}\right)}{2}=Q_{m r s}-\frac{x_{r s}}{2}$ 
The second situation, the following relationships are evident:

$n_{1 r}=\frac{Q_{m r s}}{\bar{D}_{r}} \quad$ and $\quad N=\frac{x_{r s}}{\bar{D}_{r}} \quad$ then $\quad \frac{n_{1 r}}{N}=\frac{Q_{m r s}}{x_{r s}}$

hence, $\quad \bar{I}=\frac{Q_{m r s}}{2}\left(\frac{n_{1 r}}{N}\right) \quad$ and $\quad \bar{I}=\frac{Q_{m r s}{ }^{2}}{2 x_{r s}}$

Thus, the expected average amount of inventory is given by

$\bar{I}=\int_{x_{r s}=0}^{Q_{m r s}}\left(Q_{m r s}-\frac{x_{r s}}{2}\right) f\left(x_{r s}\right) d x_{r s}+\int_{x=Q_{m r s}}^{\infty} \frac{Q_{m r s}{ }^{2}}{2 x_{r s}} f\left(x_{r s}\right) d x_{r s}$

The varying expected varying holding cost for the cycle becomes

$$
\begin{array}{r}
E\left(H C_{r s}\right)=C_{h r} N^{-\beta_{r}}\left(\int_{x_{r s}=0}^{Q_{m r s}}\left(Q_{m r s}-\frac{x_{r s}}{2}\right) f\left(x_{r s}\right) d x_{r s}\right. \\
\left.+\int_{x=Q_{m r s}}^{\infty} \frac{Q_{m r s}{ }^{2}}{2 x_{r s}} f\left(x_{r s}\right) d x_{r s}\right)
\end{array}
$$

The expected varying backlogged cost for the cycle is given by $\quad E\left(B C_{r s}\right)=C_{b r s} N^{\beta_{r}} \bar{S}$

where $\bar{S}$ represents the expected average backlogged.

In the first situation of Figure (2), the average backlogged is given by $\quad \bar{S}=0$

In the second situation, the following relationship is evident:

$n_{2 r}=\frac{x_{r s}-Q_{m r s}}{\bar{D}_{r}} \quad$ Then, $\quad \frac{n_{2 r}}{N}=\frac{x_{r s}-Q_{m r s}}{x_{r s}}$

$\bar{S}=\frac{x_{r s}-Q_{m r s}}{2}\left(\frac{n_{2 r}}{N}\right)=\frac{\left(x_{r s}-Q_{m r s}\right)^{2}}{2 x_{r s}}$

Thus, the expected average backlogged is

$\bar{S}=\int_{x=Q_{m r s}}^{\infty} \frac{\left(x_{r s}-Q_{m r s}\right)^{2}}{2 x_{r s}} f\left(x_{r s}\right) d\left(x_{r s}\right)$

The expected varying backlogged cost for the cycle becomes

$=C_{b r s} N^{\beta_{r}} \int_{x=Q_{m r s}}^{\infty} \frac{\left(x_{r s}-Q_{m r s}\right)^{2}}{2 x_{r s}} f\left(x_{r s}\right) d\left(x_{r s}\right)$

Then, the expected total cost of the cycle for multi-item multi-source is the sum of Equations (1), (2), (3) and (4) 


$$
\begin{aligned}
& E(T C) \\
& =\sum_{r=1}^{n}\left[C_{p r s} \bar{x}_{r s}+C_{o r s}+C_{h r} N^{-\beta_{r}}\left(\int_{x=0}^{Q_{m r s}}\left(Q_{m r s}-\frac{x_{r s}}{2}\right) f\left(x_{r s}\right) d x_{r s}+\int_{x=Q_{m r s}}^{\infty} \frac{Q_{m r s}{ }^{2}}{2 x_{r s}} f\left(x_{r s}\right) d x_{r s}\right)\right. \\
& \left.+C_{b r s} N^{\beta r} \int_{x=Q_{m r s}}^{\infty} \frac{\left(x_{r s}-Q_{m r s}\right)^{2}}{2 x_{r s}} f\left(x_{r s}\right) d\left(x_{r s}\right)\right]
\end{aligned}
$$

There is a limitation on the available expected varying holding cost;

$$
E(H C(N))=\sum_{r=1}^{n} C_{h r} N^{-\beta_{r}} \bar{I} \leq K_{h r s}
$$

The problem is to find the optimal maximum inventory level for a given $N$ which minimize the expected annual average total cost function (5) subject to the expected varying holding cost restriction. It may be written as

$\operatorname{Min} E\left(T C\left(, Q_{m r s}, N\right)\right)$

for all

$$
r=1,2, \ldots, n, s=1,2, \ldots, m
$$

subject to inequality constraint

$$
E(H C(N)) \leq k_{h r s}
$$

To find the optimal values $Q_{m r s}^{*}$ for a given $N$ which minimize Equation (6) under the constraint (7), the Lagrange multipliers technique with the Kuhn-Tacker conditions is used, then the Lagrange function is given by:-

$$
\begin{aligned}
\left.L\left(Q_{m s}, N\right)\right)=C_{p r s} \bar{x}_{r s} & +C_{o r s}+C_{h r} N^{-\beta_{r}}\left(\int_{x=0}^{Q_{m r s}}\left(Q_{m r s}-\frac{x_{r s}}{2}\right) f\left(x_{r s}\right) d x_{r s}+\int_{x=Q_{m r s}}^{\infty} \frac{Q_{m r s}{ }^{2}}{2 x_{r s}} f\left(x_{r s}\right) d x_{r s}\right) \\
& +C_{b r s} N^{\beta_{r}} \int_{x=Q_{m r s}}^{\infty} \frac{\left(x_{r s}-Q_{m r s}\right)^{2}}{2 x_{r s}} f\left(x_{r s}\right) d\left(x_{r s}\right) \\
& +\lambda_{h r s}\left(C_{h r} N^{-\beta_{r}}\left\{\int_{x=0}^{Q_{m r s}}\left(Q_{m r s}-\frac{x_{r s}}{2}\right) f\left(x_{r s}\right) d x_{r s} \int_{x=Q_{m r s}}^{\infty} \frac{Q_{m r s}{ }^{2}}{2 x_{r s}} f\left(x_{r s}\right) d x_{r s}\right\}\right. \\
& \left.-K_{h r s}\right)
\end{aligned}
$$

where $\lambda_{h r}$, is the Lagrange multiplier.

The optimal values $Q_{m r s}^{*}$ can be calculated by setting the corresponding first partial derivatives of Equation (8) equal to zero, and then the following equations are obtained.

$$
\begin{aligned}
\left.\frac{\partial E\left(T C\left(Q_{m r s}, N\right)\right)}{\partial Q_{m r s}}\right|_{Q_{m r s}=Q_{m r s}^{*}}=0 \\
=\frac{C_{b r s} N^{\beta_{r}}}{C_{b r s} N^{\beta_{r}}+\left(1+\lambda_{h r s}\right) C_{h r} N^{-\beta_{r}}}
\end{aligned}
$$$$
\text { hence, } \int_{x_{r s}=0}^{Q_{m r s}^{*}} f\left(x_{r s}\right) d x_{r s}+\int_{x_{r s}=Q_{m r s}^{*}}^{\infty} \frac{Q_{m r s}^{*}}{x_{r s}} f\left(x_{r s}\right) d x_{r s}
$$ 


\section{Special cases:}

Unconstrained unconstraint single-item single source model when $\beta_{r}=0, \lambda_{\text {hrs }}=0$

$$
=\frac{C_{s}}{C_{s}+C_{h}}
$$

$$
\int_{x=0}^{Q_{m}^{*}} f(x) d x+\int_{x=Q_{m}}^{\infty} \frac{Q_{m}^{*}}{x} f(x) d x
$$

Where $C_{s}$ the shortage cost per unit item. (Fabrycky W. J. and Banks Jerry [7]).

\section{The Model when all parameters are fuzzy numbers}

The inventory cost coefficients, elasticity parameters and other coefficients in the model are fuzzy in nature. Therefore, the decision variable and the objective function should be fuzzy as well, it should find the right and the left shape functions of the objective function and decision variable, by find the upper bound and the lower bound of the objective function, i.e. $\tilde{L}^{L}(\propto)$ and $\tilde{L}^{R}(\propto)$. Recall that $\tilde{L}^{L}(\propto)$ and $\tilde{L}^{R}(\propto)$ represents the largest and the smallest values (The left and right $\propto$ cuts) of the optimal objective function $\tilde{L}(\propto)$. Using approximated value of TFN which observe in Figure 3

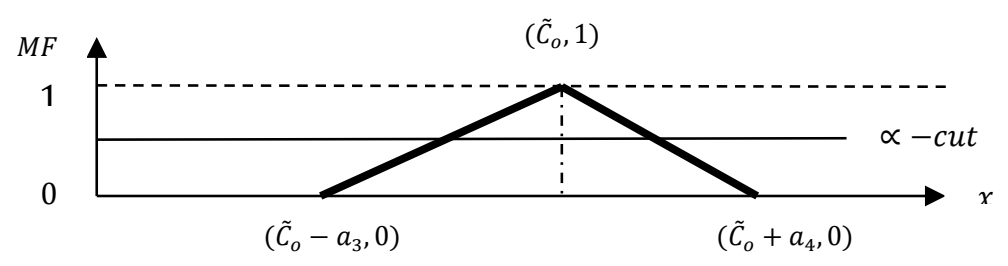

Figure 3: Order cost as triangular fuzzy number

Consider the model when all parameters are triangular fuzzy numbers (TFN) as given below

$$
\begin{array}{ll}
C_{p r s}=\left(C_{p r}-a_{1 r}, C_{p r s}, C_{p r s}+a_{2 r}\right), & C_{\text {ors }}=\left(C_{\text {ors }}-a_{3 r}, C_{\text {ors }}, C_{\text {ors }}+a_{4 r}\right), \\
C_{h r}=\left(C_{h r}-a_{5 r}, C_{h r}, C_{h r}+a_{6 r}\right), & \text { and } \quad C_{b r s}=\left(C_{b r s}-a_{7 r}, C_{b r s}, C_{b r s}+a_{8 r}\right),
\end{array}
$$

where $a_{i r}, i=1,2, \ldots \ldots, 8$ are arbitrary positive numbers under the following restrictions:

$0 \leq a_{1 r} \leq C_{p r s}, a_{2 r} \geq 0 \quad, \quad 0 \leq a_{3 r} \leq C_{o r s}, a_{4 r} \geq 0 \quad, \quad 0 \leq a_{5 r} \leq C_{h r}, a_{6 r} \geq 0 \quad$,and $\quad 0 \leq a_{7 r} \leq C_{b r s}, a_{8 r} \geq$ 0 ,

The left and right limits of $\propto$ cuts of $C_{p r s}, C_{o r s}, C_{h r}$ and $C_{b r s}$ are given by

$$
\begin{array}{ll}
\tilde{C}_{p r s_{L}}(\propto)=C_{p r s}-(1-\propto) a_{1 r}, & \tilde{C}_{p r s_{R}}(\propto)=C_{p r s}+(1-\propto) a_{2 r} \\
\tilde{C}_{o r s_{L}}(\propto)=C_{o r s}-(1-\propto) a_{3 r,} & \tilde{C}_{o r s_{R}}(\propto)=C_{o r s}+(1-\propto) a_{4 r} \\
\tilde{C}_{h r_{L}}(\propto)=C_{h r}-(1-\propto) a_{5 r}, & \tilde{C}_{h r_{R}}(\propto)=C_{h r}+(1-\propto) a_{6 r}
\end{array}
$$

and

$$
\tilde{C}_{b r s_{L}}(\propto)=C_{b r s}-(1-\propto) a_{7 r}
$$$$
\tilde{C}_{b r s_{R}}(\propto)=C_{b r s}+(1-\propto) a_{8 r}
$$ 
where

$$
\tilde{C}_{p r s}=C_{p r s}+\frac{1}{4}\left(a_{2 r}-a_{1 r}\right), \quad \tilde{C}_{\text {ors }}=C_{\text {ors }}+\frac{1}{4}\left(a_{4 r}-a_{3 r}\right),
$$

$$
\tilde{C}_{h r}=C_{h r}+\frac{1}{4}\left(a_{6 r}-a_{5 r}\right) \quad \text { and } \quad \tilde{C}_{b r s}=C_{b r s}+\frac{1}{4}\left(a_{8 r}-a_{7 r}\right)
$$

Likewise, the same steps as in crisp case will be applied here with replacing $C_{p r s}, C_{o r s}, C_{h r}$ and $C_{b r s}$ by $\tilde{C}_{p r s}, \tilde{C}_{o r s}, \tilde{C}_{h r}$ and $\tilde{C}_{b r s}$. then the optimal value of $Q_{m r s}^{*}$ for a given $N$ which minimize expected annual total cost for fuzzy case can be calculated easily and the optimal value $Q_{m r s}^{*}$ for fuzzy case can be calculated by the following equations:

$$
=\frac{\tilde{C}_{b r s} N^{\beta_{r}}}{\tilde{C}_{b r s} N^{\beta_{r}}+\left(1+\lambda_{h r s}\right) \tilde{C}_{h r} N^{-\beta_{r}}}
$$

$$
\int_{x_{r s}=0}^{Q_{m r s}^{*}} f\left(x_{r s}\right) d x_{r s}+\int_{x_{r s}=Q_{m r s}^{*}}^{\infty} \frac{Q_{m r s}}{x_{r s}} f\left(x_{r s}\right) d x_{r s}
$$

\section{The model with some continuous distributions}

Suppose that the demand for a particular item follows some continuous distribution such as:

5.1 The model with two-parameters exponential distribution: If the demand follows the two parameter exponential distribution then,

$f\left(x_{r s}\right)=\theta e^{-\theta\left(x_{r s}-\gamma\right)}, \quad \gamma<x_{r s}<\infty, \quad \theta>0$

$\theta$ continuous inverse scale parameter $\theta>0, \gamma$ continuous location parameter.

Hence, the optimal value $Q_{m r s}^{*}$ can be calculated by the following equation

$\theta\left(\int_{x_{r s}=\gamma}^{Q_{m r s}^{*}} e^{-\theta\left(x_{r s}-\gamma\right)} d x_{r s}+\int_{x_{r s}=Q_{m r s}^{*}}^{\infty} \frac{Q_{m r s}}{x_{r s}} e^{-\theta\left(x_{r s}-\gamma\right)} d x_{r s}\right)=\frac{C_{b r s} N^{\beta_{r}}}{C_{b r s} N^{\beta_{r}}+\left(1+\lambda_{h r s}\right) C_{h r} N^{-\beta_{r}}}$

5.2 The model with Kumaraswamy distribution: If the demand follows the Kumaraswamy distribution then,

$f\left(x_{r s}\right)=\frac{\alpha_{1} \alpha_{2}\left(\frac{x_{r s}-a}{b-a}\right)^{\alpha_{1}-1}\left(\left(\frac{x_{r s}-a}{b-a}\right)^{\alpha_{1}}\right)^{\alpha_{2}-1}}{b-a}, \quad a \leq x_{r s} \leq b$,

$\alpha_{1}, \alpha_{2}$ continuous shape parameter $\alpha_{1}, \alpha_{2}>0, a, b$ continuous boundary parameters $a<b$.

Hence, the optimal value $Q_{m r s}^{*}$ can be calculated by the following equation

$$
\begin{gathered}
\frac{\alpha_{1} \alpha_{2}}{b-a}\left[\int_{x_{r s}=a}^{Q_{m r s}^{*}}\left(\frac{x_{r s}-a}{b-a}\right)^{\alpha_{1}-1}\left(\left(\frac{x_{r s}-a}{b-a}\right)^{\alpha_{1}}\right)^{\alpha_{2}-1} d x_{r s}+\int_{x_{r s}=Q_{m r s}^{*}}^{b} \frac{Q_{m r s}}{x_{r s}}\left(\frac{x_{r s}-a}{b-a}\right)^{\alpha_{1}-1}\left(\left(\frac{x_{r s}-a}{b-a}\right)^{\alpha_{1}}\right)^{\alpha_{2}-1} d x_{r s}\right] \\
=\frac{C_{b r s} N^{\beta_{r}}}{C_{b r s} N^{\beta_{r}}+\left(1+\lambda_{h r s}\right) C_{h r} N^{-\beta_{r}}}
\end{gathered}
$$


5.3 The model with Gamma distribution: If the demand follows the Gamma distribution then,

$f\left(x_{r s}\right)=\frac{\left(x_{r s}-\delta\right)^{\alpha-1}}{\sigma^{\alpha} \Gamma[\alpha]} e^{\frac{-\left(x_{r s}-\delta\right)}{\sigma}}, \delta \leq x_{r s}<+\infty$

$\alpha$ Continuous shape parameter $\alpha>0, \sigma$ Continuous scale parameter $\sigma>0$

$\delta$ Continuous location parameter.

Hence, the optimal value $Q_{m r s}^{*}$ can be calculated by the following equation

$$
\int_{x_{r s}=\gamma}^{Q_{m r s}^{*}} \frac{\left(x_{r s}-\delta\right)^{\alpha-1}}{\sigma^{\alpha} \Gamma[\alpha]} e^{\frac{-\left(x_{r s}-\delta\right)}{\sigma}} d x_{r s}+\int_{x_{r s}=Q_{m r s}^{*}}^{\infty} \frac{Q_{m r s}}{x_{r s}} \frac{\left(x_{r s}-\delta\right)^{\alpha-1}}{\sigma^{\alpha} \Gamma[\alpha]} e^{\frac{-\left(x_{r s}-\delta\right)}{\sigma}} d x_{r s}=\frac{C_{b r s} N^{\beta_{r}}}{C_{b r s} N^{\beta_{r}}+\left(1+\lambda_{h r s}\right) C_{h r} N^{-\beta_{r}}}
$$

5.4 The model with Beta distribution: If the demand follows the Beta distribution then,

$\mathrm{f}\left(x_{r s}\right)=\frac{1}{\operatorname{Beta}\left[\alpha_{1}, \alpha_{2}\right]} \frac{\left(x_{r s}-\mathrm{a}\right)^{\alpha_{1}-1}\left(\mathrm{~b}-x_{r s}\right)^{\alpha_{2}-1}}{(\mathrm{~b}-\mathrm{a})^{\alpha_{1}+\alpha_{2}-1}}, a \leq x_{r s} \leq b$

$\alpha_{1}, \alpha_{2}$ Continuous shape parameter $\alpha_{1}, \alpha_{2}>0, a$ and $b$ Continuous boundary parameters

$a<b$. Hence, the optimal value $Q_{m r s}^{*}$ can be calculated by the following equation

$$
\begin{gathered}
\int_{x_{r s}=a}^{Q_{m r s}^{*}} \frac{1}{\operatorname{Beta}\left[\alpha_{1}, \alpha_{2}\right]} \frac{\left(x_{r s}-\mathrm{a}\right)^{\alpha_{1}-1}\left(\mathrm{~b}-x_{r s}\right)^{\alpha_{2}-1}}{(\mathrm{~b}-\mathrm{a})^{\alpha_{1}+\alpha_{2}-1}} d x_{r s}+\int_{x_{r s}=Q_{m r s}^{*}}^{b} \frac{Q_{m r s}}{x_{r s}} \frac{1}{\operatorname{Beta}\left[\alpha_{1}, \alpha_{2}\right]} \frac{\left(x_{r s}-\mathrm{a}\right)^{\alpha_{1}-1}\left(\mathrm{~b}-x_{r s}\right)^{\alpha_{2}-1}}{(\mathrm{~b}-\mathrm{a})^{\alpha_{1}+\alpha_{2}-1}} d x_{r s} \\
=\frac{C_{b r s} N^{\beta_{r}}}{C_{b r s} N^{\beta_{r}}+\left(1+\lambda_{h r s}\right) C_{h r} N^{-\beta_{r}}}
\end{gathered}
$$

5.5 The model with Raylieph distribution: If the demand follows the Raylieph distribution then,

$\mathrm{f}\left(x_{r s}\right)=\left(\frac{x_{r s}-\mathrm{a}}{\alpha_{2}^{2}}\right) e^{\frac{-1}{2}\left(\frac{x_{r s}-\mathrm{a}}{\alpha_{2}}\right)^{2}}, \quad \mathrm{a} \leq x_{r s}<+\infty$

$\alpha_{2}$ continuous shape parameter $\alpha_{2}>0$, a continuous location parameter.

Hence, the optimal value $Q_{m r s}^{*}$ can be calculated by the following equation

$$
\int_{x_{r s}=\gamma}^{Q_{m r s}^{*}}\left(\frac{x_{r s}-\mathrm{a}}{\alpha_{2}{ }^{2}}\right) e^{\frac{-1}{2}\left(\frac{x_{r s}-\mathrm{a}}{\alpha_{2}}\right)^{2}} d x_{r s}+\int_{x_{r s}=Q_{m r s}^{*}}^{\infty} \frac{Q_{m r s}}{x_{r s}}\left(\frac{x_{r s}-\mathrm{a}}{\alpha_{2}{ }^{2}}\right) e^{\frac{-1}{2}\left(\frac{x_{r s}-\mathrm{a}}{\alpha_{2}}\right)^{2}} d x_{r s}=\frac{C_{b r s} N^{\beta_{r}}}{C_{b r s} N^{\beta_{r}}+\left(1+\lambda_{h r s}\right) C_{h r} N^{-\beta_{r}}}
$$

5.6 The model with Erlang exponential distribution: If the demand follows the Erlang distribution then,

$$
\mathrm{f}\left(x_{r s}\right)=\frac{\left(x_{r s}-\mathrm{a}\right)^{\mathrm{m}-1}}{b^{m} \Gamma[m]} e^{\frac{-\left(x_{r s}-\mathrm{a}\right)}{b}}, \quad \mathrm{a} \leq x_{r s}<+\infty,
$$

$\mathrm{m}$ shape parameter (positive integer), $\mathrm{b}$ continuous scale parameter $\mathrm{b}>0$

a continuous location. Hence, the optimal value $Q_{m r s}^{*}$ can be calculated by the following equation 
$\int_{x_{r s}=\gamma}^{Q_{m r s}^{*}} \frac{\left(x_{r s}-\mathrm{a}\right)^{\mathrm{m}-1}}{b^{m} \Gamma[m]} e^{\frac{-\left(x_{r s}-\mathrm{a}\right)}{b}} d x_{r s}+\int_{x_{r s}=Q_{m r s}^{*}}^{\infty} \frac{Q_{m r s}}{x_{r s}} \frac{\left(x_{r s}-\mathrm{a}\right)^{\mathrm{m}-1}}{b^{m} \Gamma[m]} e^{\frac{-\left(x_{r s}-\mathrm{a}\right)}{b}} d x_{r s}=\frac{C_{b r s} N^{\beta_{r}}}{C_{b r s} N^{\beta_{r}}+\left(1+\lambda_{h r s}\right) C_{h r} N^{-\beta_{r}}}$

\section{Numerical example}

To illustrate the above developed model, consider a hypothetical inventory system with the following parameter values which are given in Table 1. It is desired to determine to optimal value $\mathrm{Q}_{\mathrm{mrs}}{ }^{*}$ which minimize the expected total cost for $\beta_{\mathrm{r}}$ between $(0,1)$ and $N=1$ month $(0.08333$ year). Also, the optimal solutions of the crisp environment and triangular fuzzy number TFN are given in Tables 3, 5, 7, 9, 11 and 13, and show by Figure 4, 5, $6,7,8$ and 9 . When the demand is follows:

1. Two parameter exponential distribution for $\gamma<x_{r s}<\infty$. at $\theta=0.20755 ; \gamma=1.6295$

2. Kumaraswamy distribution, $a \leq x_{r s} \leq b$. at $\alpha_{1}=1.5 ; \alpha_{2}=2 ; \mathrm{a}=0 ; \mathrm{b}=1$

3. Gamma distribution, $\delta \leq x_{r s}<+\infty$. at $\alpha=3 ; \delta=0.041 ; \sigma=1.3162$

4. Beta distribution, $\quad a \leq x_{r s} \leq b$. at $\alpha_{1}=1.5 ; \alpha_{2}=2 ; \mathrm{a}=0 ; \mathrm{b}=1$

5. Rayleigh distribution, $0 \leq x_{r s} \leq \infty$. at $\alpha_{2}=2 ; \mathrm{a}=0$

6. Erlang distribution, $a \leq x_{r s}<+\infty$, at ; $\mathrm{m}=2.5 ; \mathrm{a}=0 ; \mathrm{b}=1$

Table 1: Crisp and fuzzy values of the parameters

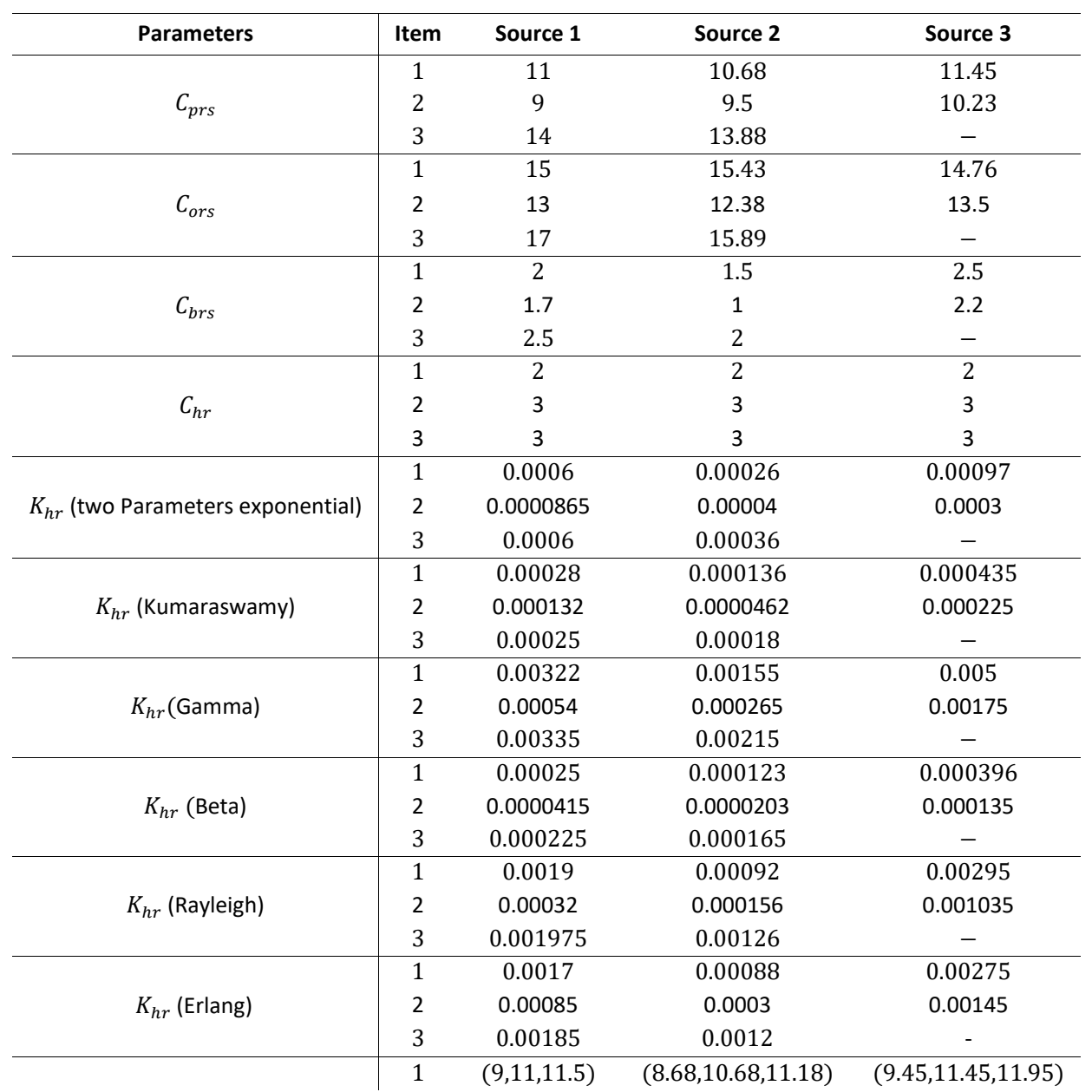




\begin{tabular}{c|cccc} 
& 2 & $(7,9,9.5)$ & $(7.5,9.5,10)$ & $(8,23,10.23,10.73)$ \\
& 3 & $(12,14,14.5)$ & $(11.88,13.88,14.38)$ & - \\
\hline \multirow{2}{*}{$\tilde{C}_{\text {prs }}$} & 1 & $(12,15,16.3)$ & $(12.43,15.4316 .73)$ & $(11.76,14.76,16.6)$ \\
& 2 & $10,13,14.3)$ & $(8.38,12.38,13,68)$ & $(10.5,13.5,14.8)$ \\
& 3 & $(14,17,18,3)$ & $12.89,15.89,17,19)$ & - \\
\hline \multirow{2}{*}{$\tilde{C}_{b r s}$} & 1 & $(1.6,2,2.2)$ & $(1.1,1.5,1.7)$ & $(2.1,2.5,2.7)$ \\
& 2 & $(0.6,1,1.2)$ & $(0.3,0.7,0.9)$ & $(1.4,1.8,2)$ \\
& 3 & $2.1,2.5,2.7)$ & $(1.6,2,2.2)$ & - \\
\hline \multirow{2}{*}{$\tilde{C}_{h r}$} & 1 & $(1.7,2,2.1)$ & $(1.7,2,2.1)$ & $(1.7,2,2.1)$ \\
& 2 & $(2.7,3,3.1)$ & $(2.7,3,3.1)$ & $(2.7,3,3.1)$ \\
$\widetilde{K}_{h r}$ (two Parameter exponential) & 3 & $(2.7,3,3.1)$ & $(2.7,3,3.1)$ & $(2.7,3,3.1)$ \\
\hline \multirow{3}{*}{$\widetilde{K}_{h r}$ (Kumaraswamy) } & 1 & 0.00057 & 0.00031 & 0.00096 \\
& 2 & 0.000075 & 0.000035 & 0.00027 \\
& 3 & 0.0006 & 0.00036 & - \\
\hline \multirow{2}{*}{$\widetilde{K}_{h r}$ (Gamma) } & 1 & 0.000265 & 0.00013 & 0.00041 \\
& 2 & 0.0000424 & 0.0000195 & 0.000142 \\
& 3 & 0.00028 & 0.00015 & - \\
\hline \multirow{3}{*}{$\widetilde{K}_{h r}$ (Beta) } & 1 & 0.003 & 0.00172 & 0.0049 \\
& 2 & 0.0005 & 0.0002 & 0.00165 \\
& 3 & 0.00325 & 0.002 & - \\
\hline \multirow{2}{*}{$\widetilde{K}_{h r}$ (Erlang) } & 1 & 0.00024 & 0.000132 & 0.00035 \\
& 2 & 0.0000382 & 0.0000178 & 0.00012 \\
& 3 & 0.000256 & 0.000162 & - \\
\hline & 1 & 0.0018 & 0.001035 & 0.0029 \\
& 2 & 0.000295 & 0.000138 & 0.000995 \\
& 3 & 0.00192 & 0.001235 & - \\
\hline & 1 & 0.00174 & 0.00084 & 0.0027 \\
& 2 & 0.00083 & 0.000277 & 0.0014 \\
& 3 & 0.00182 & 0.00182 & - \\
\hline & & & & \\
\hline
\end{tabular}

Table 2: The results of crisp and fuzzy values for two parameters exponential distribution

\begin{tabular}{|c|c|c|c|c|c|c|c|c|c|c|}
\hline \multirow{2}{*}{$\beta_{r}$} & \multirow[t]{2}{*}{$\sim$} & \multicolumn{3}{|c|}{ Item 1} & \multicolumn{3}{|c|}{ Item 2} & \multicolumn{3}{|c|}{ Item 3} \\
\hline & & $\lambda_{h r}$ & $Q_{m 1}$ & $E(T C)_{1}$ & $\lambda_{h r}$ & $Q_{m 2}$ & $E(T C)_{2}$ & $\lambda_{h r}$ & $Q_{m 3}$ & $E(T C)_{3}$ \\
\hline \multirow{3}{*}{0.1} & 1 & 20.2256 & 0.0155365 & 20.2377 & 34.7972 & 0.0044178 & 17.4717 & 20.3643 & 0.0124822 & 23.555 \\
\hline & 2 & 20.9304 & 0.0098981 & 19.1046 & 29.1793 & 0.0029292 & 15.012 & 20.6788 & 0.0094812 & 21.1403 \\
\hline & 3 & 20.2076 & 0.0201511 & 21.2952 & 24.9585 & 0.0085965 & 19.2772 & - & - & - \\
\hline \multirow{3}{*}{0.2} & 1 & 13.4974 & 0.0135852 & 19.0887 & 23.4535 & 0.0038694 & 16.4886 & 13.591 & 0.0109182 & 22.1162 \\
\hline & 2 & 13.9773 & 0.0086608 & 18.2976 & 19.6206 & 0.0025667 & 14.4332 & 13.8055 & 0.0082964 & 19.9872 \\
\hline & 3 & 13.4877 & 0.0176127 & 19.8628 & 16.7285 & 0.0075233 & 18.0082 & - & - & - \\
\hline \multirow{3}{*}{0.3} & 1 & 8.90146 & 0.0118814 & 18.1914 & 15.7056 & 0.0033896 & 15.7216 & 8.96493 & 0.009552 & 20.9929 \\
\hline & 2 & 9,22886 & 0.0075795 & 17..6677 & 13.0905 & 0.0022493 & 13.9817 & 9.11159 & 0.0072611 & 19.0871 \\
\hline & 3 & 8.89603 & 0.0153975 & 18.7439 & 11.1081 & 0.0065852 & 17.0177 & - & - & - \\
\hline \multirow{3}{*}{0.4} & 1 & 5.76228 & 0.0103934 & 17.4909 & 10.4134 & 0.00296962 & 15.1232 & 5.80554 & 0.0083583 & 20.116 \\
\hline & 2 & 5.98603 & 0.0066343 & 17.1761 & 8.62923 & 0.00197143 & 13.6294 & 5.90601 & 0.006356 & 18.3847 \\
\hline & 3 & 5.02039 & 0.0134639 & 17.8679 & 6.36613 & 0.00576506 & 16.2439 & - & - & - \\
\hline \multirow{3}{*}{0.5} & 1 & 3.61827 & 0.0090936 & 16.944 & 6.79838 & 0.00260207 & 14.6563 & 3.64791 & 0.0073151 & 19.4316 \\
\hline & 2 & 3.77144 & 0.005808 & 16.7924 & 5.58101 & 0.0017281 & 13.3546 & 3.71685 & 0.0055646 & 17.8365 \\
\hline & 3 & 3.61631 & 0.0117755 & 17.1876 & 4.64854 & 0.00504787 & 15.6415 & - & - & - \\
\hline \multirow{3}{*}{0.6} & 1 & 2.15406 & 0.0079577 & 16.5171 & 4.32878 & 0.00228031 & 14.2921 & 2.17442 & 0.0064032 & 18.8974 \\
\hline & 2 & 2.25903 & 0.0050854 & 16.4931 & 3.49814 & 0.00151498 & 13.1403 & 2.2218 & 0.0048726 & 17.4088 \\
\hline & 3 & 2.15274 & 0.0103009 & 16.6548 & 2.85834 & 0.00442059 & 15.1708 & - & - & - \\
\hline \multirow{3}{*}{0.7} & 1 & 1.15411 & 0.006965 & 16.1839 & 2.64155 & 0.00199859 & 14.0079 & 1.16815 & 0.0056059 & 18.4806 \\
\hline & 2 & 1.22613 & 0.0044534 & 16.2594 & 2.07477 & 0.00132829 & 12.973 & 1.20075 & 0.0042672 & 17.075 \\
\hline & 3 & 1.15315 & 0.0090127 & 16.2389 & 1.63568 & 0.00387183 & 14.8036 & - & - & - \\
\hline \multirow{3}{*}{0.8} & 1 & 0.471232 & 0.0060971 & 15.9239 & 1.48877 & 0.00175188 & 13.7863 & 0.480927 & 0.0049087 & 18.1553 \\
\hline & 2 & 0.520693 & 0.0039006 & 16.0771 & 1.10198 & 0.00116474 & 12.8426 & 0.503382 & 0.0037377 & 16.8145 \\
\hline & 3 & 0.4705 & 0.0078871 & 15.9143 & 0.800586 & 0.00339168 & 14.517 & - & - & - \\
\hline \multirow{2}{*}{0.9} & 1 & 0.004876 & 0.0053382 & 15.721 & 0.701063 & 0.0015358 & 13.6133 & 0.011587 & 0.0042989 & 17.9014 \\
\hline & 2 & 0.03887 & 0.0078871 & 15.9349 & 0.437103 & 0.0010214 & 12.7409 & 0.027067 & 0.0032743 & 16.6113 \\
\hline
\end{tabular}




\begin{tabular}{|c|c|c|c|c|c|c|c|c|c|c|}
\hline & 3 & 0.004303 & 0.0069032 & 15.6609 & 0.230177 & 0.0029715 & 14.2935 & - & - & - \\
\hline$\widetilde{\boldsymbol{\beta}}_{r}$ & & $\tilde{\lambda}_{h r}$ & $\widetilde{Q}_{m 1}$ & $E(\widetilde{T C})_{1}$ & $\tilde{\lambda}_{h r}$ & $\widetilde{Q}_{m 2}$ & $E(\widetilde{T C})_{2}$ & $\tilde{\lambda}_{h r}$ & $\widetilde{Q}_{m 3}$ & $E(\widetilde{T C})_{3}$ \\
\hline \multirow{3}{*}{0.1} & 1 & 20.484 & 0.0153199 & 19.6822 & 20.5729 & 0.0041309 & 15.0742 & 20.126 & 0.0125958 & 22.9986 \\
\hline & 2 & 20.2135 & 0.01103 & 18.8091 & 20.0671 & 0.0027529 & 13.666 & 20.3276 & 0.0095672 & 20.5839 \\
\hline & 3 & 20.1681 & 0.0203152 & 20.7391 & 20.8823 & 0.0081974 & 17.6713 & - & - & - \\
\hline \multirow{3}{*}{0.2} & 1 & 13.6738 & 0.0133961 & 18.5617 & 13.7372 & 0.0036184 & 14.5248 & 13.4283 & 0.0110174 & 21.5887 \\
\hline & 2 & 13.4878 & 0.0096496 & 17.9739 & 13.3951 & 0.0024123 & 13.2897 & 13.5657 & 0.0083716 & 19.4596 \\
\hline & 3 & 13.4608 & 0.0177559 & 19.3355 & 13.9448 & 0.0071744 & 16.6616 & - & - & - \\
\hline \multirow{3}{*}{0.3} & 1 & 9.02189 & 0.0117163 & 17.6868 & 9.06821 & 0.0031699 & 14.0961 & 8.85377 & 0.0096387 & 20.4879 \\
\hline & 2 & 8.89443 & 0.0084436 & 17.3219 & 8.8369 & 0.0021142 & 12.9962 & 8.94777 & 0.0073268 & 18.5821 \\
\hline & 3 & 8.8777 & 0.0155225 & 18.239 & 9.207 & 0.0062802 & 15.8735 & - & - & - \\
\hline \multirow{3}{*}{0.4} & 1 & 5.8445 & 0.0102492 & 17.0037 & 5.87896 & 0.0027774 & 13.7616 & 5.72963 & 0.0084341 & 19.6286 \\
\hline & 2 & 5.75748 & 0.0073897 & 16.813 & 5.72264 & 0.0018531 & 12.7672 & 5.79411 & 0.0064134 & 17.8973 \\
\hline & 3 & 5.74661 & 0.013573 & 17.3827 & 5.97147 & 0.0054984 & 15.2586 & - & - & - \\
\hline \multirow{3}{*}{0.5} & 1 & 3.67444 & 0.0089675 & 16.4705 & 3.70034 & 0.0024338 & 13.5007 & 3.59605 & 0.0073814 & 18.9579 \\
\hline & 2 & 3.6152 & 0.0064684 & 16.4158 & 3.59471 & 0.0016245 & 12.5886 & 3.64041 & 0.0056148 & 17.3628 \\
\hline & 3 & 3.60779 & 0.0118708 & 16.714 & 3.76182 & 0.0048146 & 14.7787 & - & - & - \\
\hline \multirow{3}{*}{0.6} & 1 & 2.19242 & 0.0078476 & 16.0542 & 2.21196 & 0.002133 & 13.2971 & 2.139 & 0.0064611 & 18.4344 \\
\hline & 2 & 2.15219 & 0.005663 & 16.1058 & 2.14064 & 0.0014242 & 12.4492 & 2.16957 & 0.0049165 & 16.9458 \\
\hline & 3 & 2.14691 & 0.0103842 & 16.1919 & 2.25272 & 0.0042166 & 14.4042 & - & - & - \\
\hline \multirow{3}{*}{0.7} & 1 & 1.18032 & 0.0068687 & 15.7294 & 1.19508 & 0.0018696 & 13.1383 & 1.14395 & 0.0056566 & 18.0259 \\
\hline & 2 & 1.15306 & 0.0049586 & 15.8639 & 1.14691 & 0.0012488 & 12.3405 & 1.16506 & 0.0043057 & 16.6203 \\
\hline & 3 & 1.14918 & 0.0090854 & 15.7843 & 1.22202 & 0.0036933 & 14.112 & - & - & - \\
\hline \multirow{3}{*}{0.8} & 1 & 0.48914 & 0.0060129 & 15.4758 & 0.518034 & 0.0032355 & 13.8841 & 0.46439 & 0.004953 & 17.7072 \\
\hline & 2 & 0.4707 & 0.0043425 & 15.6752 & 0.467747 & 0.0010951 & 12.2557 & 0.478999 & 0.0037713 & 16.3664 \\
\hline & 3 & 0.46779 & 0.0079506 & 15.4662 & 0.478999 & 0.0037713 & 16.3664 & - & - & - \\
\hline \multirow{3}{*}{0.9} & 1 & 0.017111 & 0.0052646 & 15.278 & 0.02547 & 0.0014368 & 12.9178 & 0.00029 & 0.0043376 & 17.4584 \\
\hline & 2 & 0.00466 & 0.0038035 & 15.5279 & 0.003523 & 0.0009604 & 12.1896 & 0.010404 & 0.0033037 & 16.1683 \\
\hline & 3 & 0.00245 & 0.0153199 & 15.2179 & 0.037163 & 0.0028348 & 13.7062 & - & - & - \\
\hline
\end{tabular}

Table 3: The Optimal policy variable for two parameter exponential distribution at $\beta=0.9$

\begin{tabular}{ccccccc}
\hline Item & source & $Q^{*}{ }_{m r s}$ & Min E(TC) & $\widetilde{Q}^{*}{ }_{m r s}$ & Source & Min E( $\widetilde{\mathrm{TC}})$ \\
\hline 1 & 3 & 0.00690321 & & 0.00695875 & 3 & \\
2 & 2 & 0.00102143 & 45.0131 & 0.000960373 & 2 & 43.5758 \\
3 & 2 & 0.00327427 & & 0.0033037 & 2 & \\
\hline
\end{tabular}

Table 4: The results of crisp and fuzzy values for Kamurswamy distribution

\begin{tabular}{|c|c|c|c|c|c|c|c|c|c|c|}
\hline \multirow{2}{*}{$\beta_{r}$} & \multirow{2}{*}{$\sim$} & \multicolumn{3}{|c|}{ Item 1} & \multicolumn{3}{|c|}{ Item 2} & \multicolumn{3}{|c|}{ Item 3} \\
\hline & & $\lambda_{h r}$ & $Q_{m 1}$ & $E(T C)_{1}$ & $\lambda_{h r}$ & $Q_{m 2}$ & $E(T C)_{2}$ & $\lambda_{h r}$ & $Q_{m 3}$ & $E(T C)_{3}$ \\
\hline \multirow{3}{*}{0.1} & 1 & 18.7367 & 0.0071865 & 15.3403 & 18.9662 & 0.0039964 & 13.2932 & 20.3354 & 0.0055227 & 17.4284 \\
\hline & 2 & 18.8417 & 0.0049818 & 15.6705 & 18.8251 & 0.0023514 & 12.5537 & 19.1175 & 0.0046757 & 16.234 \\
\hline & 3 & 18.7635 & 0.0089916 & 15.1821 & 18.792 & 0.0052354 & 13.8775 & - & - & - \\
\hline \multirow{3}{*}{0.2} & 1 & 12.6023 & 0.0063344 & 15.2665 & 12.7508 & 0.0035244 & 13.2292 & 13.6984 & 0.0048691 & 17.3352 \\
\hline & 2 & 12.6678 & 0.0043927 & 15.6181 & 12.65 & 0.0020744 & 12.5157 & 12.8569 & 0.004123 & 16.159 \\
\hline & 3 & 12.627 & 0.0079235 & 15.0909 & 12.6343 & 0.0046161 & 13.7953 & - & - & - \\
\hline \multirow{3}{*}{0.3} & 1 & 8.37271 & 0.0055841 & 15.2086 & 8.46944 & 0.0031084 & 13.1791 & 9.12467 & 0.004293 & 17.2621 \\
\hline & 2 & 8.41382 & 0.0038736 & 15.5771 & 8.39806 & 0.0018302 & 12.4859 & 8.54353 & 0.003636 & 16.1003 \\
\hline & 3 & 8.39336 & 0.0069834 & 15.0192 & 8.39119 & 0.0040705 & 13.7309 & - & - & - \\
\hline \multirow{3}{*}{0.4} & 1 & 5.45716 & 0.0049232 & 15.1632 & 5.52063 & 0.0027418 & 13.14 & 5.97331 & 0.0037861 & 17.205 \\
\hline & 2 & 5.48319 & 0.0034162 & 15.545 & 5.47047 & 0.0016148 & 12.4627 & 5.57224 & 0.0032068 & 16.0544 \\
\hline & 3 & 5.4735 & 0.0061556 & 14.963 & 5.46785 & 0.0035897 & 13.6805 & - & - & - \\
\hline \multirow{3}{*}{0.5} & 1 & 3.44791 & 0.0043411 & 15.1277 & 3.48984 & 0.0024186 & 13.1094 & 3.80236 & 0.0033391 & 17.1602 \\
\hline & 2 & 3.46453 & 0.0030131 & 15.5199 & 3.45476 & 0.0014249 & 12.4446 & 3.5257 & 0.0028285 & 16.0185 \\
\hline & 3 & 3.46039 & 0.0054266 & 14.919 & 3.4541 & 0.003166 & 13.6411 & - & - & - \\
\hline \multirow{3}{*}{0.6} & 1 & 2.0635 & 0.0038281 & 15.0999 & 2.09138 & 0.0021337 & 13.0854 & 2.30702 & 0.0029452 & 17.1253 \\
\hline & 2 & 2.07421 & 0.0026578 & 15.5002 & 2.06697 & 0.0012574 & 12.4304 & 2.11625 & 0.0024951 & 15.9904 \\
\hline & 3 & 2.0728 & 0.0047845 & 14.8844 & 2.06712 & 0.0027926 & 13.6103 & - & - & - \\
\hline
\end{tabular}




\begin{tabular}{|c|c|c|c|c|c|c|c|c|c|c|}
\hline \multirow{3}{*}{0.7} & 1 & 1.10978 & 0.0033762 & 15.0781 & 1.12844 & 0.0018825 & 13.0667 & 1.27714 & 0.0025979 & 17.0979 \\
\hline & 2 & 1.11676 & 0.0023445 & 15.4849 & 1.1115 & 0.0011096 & 12.4194 & 1.14565 & 0.0022011 & 15.9684 \\
\hline & 3 & 1.11658 & 0.0042188 & 14.8574 & 1.1119 & 0.0024634 & 13.5862 & - & - & - \\
\hline \multirow{3}{*}{0.8} & 1 & 0.45285 & 0.0029778 & 15.0611 & 0.46541 & 0.0016609 & 13.0521 & 0.56792 & 0.0022917 & 17.0765 \\
\hline & 2 & 0.457436 & 0.0020684 & 15.4729 & 0.45371 & 0.0009792 & 12.4107 & 0.47731 & 0.0019419 & 15.9513 \\
\hline & 3 & 0.457763 & 0.0037204 & 14.8362 & 0.45412 & 0.0021731 & 13.5674 & - & - & - \\
\hline \multirow{3}{*}{0.9} & 1 & 0.00041 & 0.0026267 & 15.0478 & 0.00891 & 0.0014655 & 13.0407 & 0.07954 & 0.0020218 & 17.0598 \\
\hline & 2 & 0.003446 & 0.0018249 & 15.4635 & 0.00084 & 0.0008642 & 12.404 & 0.01829 & 0.0017113 & 15.9379 \\
\hline & 3 & 0.003917 & 0.0032812 & 14.8197 & 0.00117 & 0.0019172 & 13.5527 & - & - & - \\
\hline \multirow[t]{2}{*}{$\tilde{\beta}_{r}$} & & $\tilde{\lambda}_{h r}$ & $\tilde{Q}_{m 1}$ & $E(\widetilde{T C})_{1}$ & $\tilde{\lambda}_{h r}$ & $\tilde{Q}_{m 2}$ & $E(\widetilde{T C})_{2}$ & $\tilde{\lambda}_{h r}$ & $\tilde{Q}_{m 3}$ & $E(\widetilde{T C})_{3}$ \\
\hline & 1 & 19.0338 & 0.0070787 & 14.9069 & 18.8231 & 0.002271 & 12.7401 & 18.9211 & 0.0058995 & 16.9942 \\
\hline \multirow[t]{3}{*}{0.1} & 2 & 20.2871 & 0.0049321 & 15.2541 & 18.9682 & 0.0015353 & 12.0683 & 20.6673 & 0.0042999 & 15.8009 \\
\hline & 3 & 19.2071 & 0.0088379 & 14.7489 & 18.9848 & 0.0041822 & 13.3766 & - & - & - \\
\hline & 1 & 12.8068 & 0.0062395 & 14.8349 & 12.6485 & 0.0020035 & 12.7039 & 12.7253 & 0.005201 & 16.903 \\
\hline \multirow[t]{3}{*}{0.2} & 2 & 13.6633 & 0.0043489 & 15.1998 & 12.7476 & 0.0013547 & 12.0435 & 13.9232 & 0.0037918 & 15.7276 \\
\hline & 3 & 12.9324 & 0.0077882 & 14.6594 & 12.7641 & 0.0036882 & 13.3108 & - & - & - \\
\hline & 1 & 8.51334 & 0.0055005 & 14.7785 & 8.39698 & 0.0017676 & 12.6757 & 8.45499 & 0.0045858 & 16.8316 \\
\hline \multirow[t]{3}{*}{0.3} & 2 & 9.09936 & 0.003835 & 15.1573 & 8.46484 & 0.0011955 & 12.024 & 9.27731 & 0.0033441 & 15.6703 \\
\hline & 3 & 8.6035 & 0.0068642 & 14.5892 & 8.47886 & 0.0032528 & 13.2593 & - & - & - \\
\hline & 1 & 5.55394 & 0.0048496 & 14.7342 & 5.46966 & 0.0015597 & 12.6536 & 5.51245 & 0.0040438 & 16.7757 \\
\hline \multirow[t]{3}{*}{0.4} & 2 & 5.95527 & 0.0033822 & 15.1241 & 5.51623 & 0.001055 & 12.0089 & 6.07721 & 0.0029496 & 15.6254 \\
\hline & 3 & 5.61814 & 0.0060507 & 14.5341 & 5.52727 & 0.0028691 & 13.219 & - & - & - \\
\hline & 1 & 3.5145 & 0.0042762 & 14.6996 & 3.45419 & 0.0013762 & 12.6364 & 3.4852 & 0.0035662 & 16.7319 \\
\hline \multirow[t]{3}{*}{0.5} & 2 & 3.7896 & 0.0029831 & 15.0981 & 3.48623 & 0.0009311 & 11.9971 & 3.87322 & 0.0026018 & 15.5904 \\
\hline & 3 & 3.55993 & 0.0053342 & 14.4908 & 3.4945 & 0.0025308 & 13.1875 & - & - & - \\
\hline & 1 & 2.10932 & 0.003771 & 14.6724 & 2.06657 & 0.0012144 & 12.6229 & 2.08873 & 0.0031453 & 16.6977 \\
\hline \multirow[t]{3}{*}{0.6} & 2 & 2.29804 & 0.0026313 & 15.0777 & 2.08865 & 0.0008217 & 11.9878 & 2.35544 & 0.0022952 & 15.5629 \\
\hline & 3 & 2.14131 & 0.0047031 & 14.457 & 2.09463 & 0.0022326 & 13.1629 & - & - & - \\
\hline & 1 & 1.14131 & 0.0033258 & 14.6512 & 1.11124 & 0.0010717 & 12.6124 & 1.12691 & 0.0027743 & 16.6709 \\
\hline \multirow[t]{3}{*}{0.7} & 2 & 1.27086 & 0.0023212 & 15.0618 & 1.12647 & 0.0007253 & 11.9806 & 1.31028 & 0.0020248 & 15.5415 \\
\hline & 3 & 1.16374 & 0.0041471 & 14.4305 & 1.1307 & 0.0019697 & 13.1437 & - & - & - \\
\hline & 1 & 0.47456 & 0.0029334 & 14.6346 & 0.453528 & 0.0009458 & 12.6042 & 0.46451 & 0.0024473 & 16.65 \\
\hline \multirow[t]{2}{*}{0.8} & 2 & 0.56354 & 0.0020478 & 15.0494 & 0.46405 & 0.0006402 & 11.975 & 0.59063 & 0.0017865 & 15.5248 \\
\hline & 3 & 0.49022 & 0.0036573 & 14.4097 & 0.46698 & 0.0017378 & 13.1287 & - & - & - \\
\hline \multirow{3}{*}{0.9} & 1 & 0.01535 & 0.0025875 & 14.6216 & 0.000715 & 0.0008348 & 12.5978 & 0.00836 & 0.0021589 & 16.6336 \\
\hline & 2 & 0.07649 & 0.0018067 & 15.0397 & 0.07993 & 0.000565 & 11.9706 & 0.09512 & 0.0015763 & 15.5117 \\
\hline & 3 & 0.02626 & 0.0032255 & 14.3935 & 0.00999 & 0.0015334 & 13.1169 & - & - & - \\
\hline
\end{tabular}

Table 5: The Optimal policy variable for Kamurswamy distribution at $\beta=0.9$

\begin{tabular}{ccccccc}
\hline Item & source & $Q^{*}{ }_{m r s}$ & Min E(TC) & $\tilde{Q}^{*}{ }_{m r s}$ & Source & Min E( $\widetilde{\mathrm{TC}})$ \\
\hline 1 & 3 & 0.00690321 & & 0.00695875 & 3 & \\
2 & 2 & 0.00102143 & 45.0131 & 0.000960373 & 2 & 43.5758 \\
3 & 2 & 0.00327427 & & 0.0033037 & 2 & \\
& & & & & &
\end{tabular}

Table 6: The results of crisp and fuzzy values for Gamma distribution

\begin{tabular}{|c|c|c|c|c|c|c|c|c|c|c|}
\hline \multirow{2}{*}{$\beta_{r}$} & & \multicolumn{4}{|c|}{ Item 1} & \multicolumn{2}{|c|}{ Item 2} & \multicolumn{3}{|c|}{ Item 3} \\
\hline & & $\lambda_{h r}$ & $Q_{m 1}$ & $E(T C)_{1}$ & $\lambda_{h r}$ & $Q_{m 2}$ & $E(T C)_{2}$ & $\lambda_{h r}$ & $Q_{m 3}$ & $E(T C)_{3}$ \\
\hline \multirow{3}{*}{0.1} & 1 & 18.3718 & 0.0824827 & 17.9883 & 32.5157 & 0.0275794 & 15.6092 & 18.4852 & 0.0686927 & 20.7608 \\
\hline & 2 & 18.7322 & 0.0572268 & 17.548 & 27.2306 & 0.0193201 & 13.9211 & 18.5586 & 0.055031 & 18.919 \\
\hline & 3 & 18.283 & 0.102784 & 18.4581 & 22.8952 & 0.0496486 & 16.8403 & - & - & - \\
\hline \multirow{3}{*}{0.2} & 1 & 12.3929 & 0.0728456 & 17.3429 & 22.115 & 0.0243571 & 15.0385 & 12.4631 & 0.0606669 & 19.946 \\
\hline & 2 & 12.6267 & 0.0505406 & 17.0879 & 18.4633 & 0.0170627 & 13.5835 & 12.5055 & 0.0486013 & 18.2607 \\
\hline & 3 & 12.3438 & 0.0907741 & 17.6632 & 15.496 & 0.0438478 & 16.1134 & - & - & - \\
\hline \multirow{3}{*}{0.3} & 1 & 8.2554 & 0.0643345 & 16.836 & 14.9397 & 0.0215113 & 14.5924 & 8.2988 & 0.0535787 & 19.3068 \\
\hline & 2 & 8.40756 & 0.0446356 & 16.7273 & 12.4176 & 0.015069 & 13.3197 & 8.32308 & 0.0429229 & 17.7448 \\
\hline & 3 & 8.22885 & 0.0801681 & 17.0377 & 10.3851 & 0.0387248 & 15.544 & - & - & - \\
\hline \multirow{2}{*}{0.4} & 1 & 5.39366 & 0.0568178 & 16.4382 & 9.99044 & 0.0189979 & 14.2437 & 5.42055 & 0.0473187 & 18.8057 \\
\hline & 2 & 5.49305 & 0.0394205 & 16.4449 & 8.24915 & 0.0133082 & 13.1136 & 5.43425 & 0.0379079 & 17.3409 \\
\hline
\end{tabular}




\begin{tabular}{|c|c|c|c|c|c|c|c|c|c|c|}
\hline & 3 & 5.37984 & 0.0708014 & 16.5461 & 6.85585 & 0.0342003 & 15.0983 & - & - & - \\
\hline \multirow{3}{*}{0.5} & 1 & 3.41527 & 0.0501794 & 16.1262 & 6.57717 & 0.0167781 & 13.9713 & 3.43195 & 0.0417902 & 18.413 \\
\hline & 2 & 3.48044 & 0.0348147 & 16.2238 & 5.37539 & 0.011753 & 12.9527 & 3.43956 & 0.0334789 & 17.0246 \\
\hline & 3 & 3.40849 & 0.0625291 & 16.16 & 4.41957 & 0.0302044 & 14.7495 & - & - & - \\
\hline \multirow{3}{*}{0.6} & 1 & 2.04815 & 0.0443166 & 15.8817 & 4.22348 & 0.0148177 & 13.7584 & 2.05853 & 0.0369075 & 18.1065 \\
\hline & 2 & 2.09103 & 0.0307471 & 16.0507 & 3.39435 & 0.0103794 & 12.8271 & 2.06265 & 0.0295673 & 16.7772 \\
\hline & 3 & 2.04515 & 0.0552234 & 15.8571 & 2.73818 & 0.0266754 & 14.4767 & - & - & - \\
\hline \multirow{3}{*}{0.7} & 1 & 1.10379 & 0.0391387 & 15.6902 & 2.60068 & 0.0130862 & 13.5922 & 1.11025 & 0.0325954 & 17.8649 \\
\hline & 2 & 1.1321 & 0.0271547 & 15.9154 & 2.02883 & 0.00916609 & 12.729 & 1.11242 & 0.0261127 & 16.5837 \\
\hline & 3 & 1.10274 & 0.0487713 & 15.6196 & 1.57804 & 0.0235587 & 14.2634 & - & - & - \\
\hline \multirow{3}{*}{0.8} & 1 & 0.45167 & 0.0345659 & 15.5403 & 1.48192 & 0.0115569 & 13.4624 & 0.455714 & 0.028787 & 17.6767 \\
\hline & 2 & 0.47044 & 0.023982 & 15.8095 & 1.08763 & 0.0080944 & 12.6524 & 0.456796 & 0.0230618 & 16.4324 \\
\hline & 3 & 0.45157 & 0.043073 & 15.4335 & 0.7777 & 0.0208062 & 14.0966 & - & - & - \\
\hline \multirow{3}{*}{0.9} & 1 & 0.00149 & 0.0305273 & 15.423 & 0.7107 & 0.0102062 & 13.361 & 0.0040254 & 0.0254236 & 17.5294 \\
\hline & 2 & 0.01397 & 0.02118 & 15.7268 & 0.43893 & 0.00714771 & 12.5926 & 0.0045259 & 0.0203673 & 16. 3141 \\
\hline & 3 & 0.001794 & 0.0380405 & 15.2877 & 0.22569 & 0.0183752 & 13.9663 & - & - & - \\
\hline \multirow[t]{2}{*}{$\tilde{\beta}_{r}$} & & $\tilde{\lambda}_{h r}$ & $\tilde{Q}_{m 1}$ & $E(\widetilde{T C})_{1}$ & $\tilde{\lambda}_{h r}$ & $\tilde{Q}_{m 2}$ & $E(\widetilde{T C})_{2}$ & $\tilde{\lambda}_{h r}$ & $\tilde{Q}_{m 3}$ & $E(\widetilde{T C})_{3}$ \\
\hline & 1 & 18.8309 & 0.0806294 & 17.4912 & 18.6344 & 0.0267623 & 14.0339 & 18.5541 & 0.0682307 & 20.2615 \\
\hline \multirow[t]{2}{*}{0.1} & 2 & 18.6195 & 0.0610514 & 17.1945 & 20.32 & 0.0169258 & 12.958 & 18.95 & 0.0535245 & 18.4204 \\
\hline & 3 & 18.3305 & 0.103047 & 17.9587 & 18.7479 & 0.0486161 & 15.7337 & - & - & - \\
\hline \multirow{3}{*}{0.2} & 1 & 12.7093 & 0.0712088 & 16.8611 & 12.5409 & 0.0236354 & 13.7148 & 12.5104 & 0.0602588 & 19.4627 \\
\hline & 2 & 12.5512 & 0.0539184 & 16.7193 & 13.6975 & 0.0149481 & 12.7381 & 12.7748 & 0.0472709 & 17.7779 \\
\hline & 3 & 12.3768 & 0.0910066 & 17.1798 & 12.6323 & 0.0429359 & 15.155 & - & - & - \\
\hline \multirow{3}{*}{0.3} & 1 & 8.47332 & 0.0628889 & 16.3663 & 8.33729 & 0.0208739 & 13.4654 & 8.3313 & 0.0532183 & 18.836 \\
\hline & 2 & 8.35685 & 0.0476187 & 16.3467 & 9.13139 & 0.0132014 & 12.5664 & 8.50843 & 0.0417479 & 17.2744 \\
\hline & 3 & 8.25177 & 0.0803734 & 16.5669 & 8.40824 & 0.0379194 & 14.7018 & - & - & - \\
\hline \multirow{3}{*}{0.4} & 1 & 5.54377 & 0.05554111 & 15.978 & 5.43789 & 0.018435 & 13.2704 & 5.44288 & 0.0470005 & 18.3448 \\
\hline & 2 & 5.4589 & 0.04205511 & 16.0548 & 5.98353 & 0.0116586 & 12.4323 & 5.56183 & 0.0368702 & 16.8802 \\
\hline & 3 & 5.39574 & 0.0709827 & 16.0852 & 5.49159 & 0.033489 & 14.3471 & - & - & - \\
\hline \multirow{3}{*}{0.5} & 1 & 3.51867 & 0.0490519 & 15.6735 & 3.43839 & 0.016281 & 13.1181 & 3.44731 & 0.0415091 & 17.9599 \\
\hline & 2 & 3.45739 & 0.0371415 & 15.8263 & 3.81352 & 0.010296 & 12.3276 & 3.52738 & 0.0325624 & 16.5717 \\
\hline & 3 & 3.41951 & 0.0626893 & 15.7069 & 3.47824 & 0.0295763 & 14.0695 & - & - & - \\
\hline \multirow{3}{*}{0.6} & 1 & 2.11938 & 0.0433208 & 15.435 & 2.05965 & 0.0143786 & 12.9991 & 2.06908 & 0.0366593 & 17.6586 \\
\hline & 2 & 2.07545 & 0.032802 & 15.6473 & 2.31772 & 0.00909249 & 12.2458 & 2.12311 & 0.0287579 & 16.3303 \\
\hline & 3 & 2.05278 & 0.0553648 & 15.4101 & 2.08882 & 0.0261206 & 13.8524 & - & - & - \\
\hline \multirow{3}{*}{0.7} & 1 & 1.15286 & 0.0382593 & 15.2481 & 1.10907 & 0.0126984 & 12.9062 & 1.11752 & 0.0323761 & 17.4227 \\
\hline & 2 & 1.12155 & 0.0289695 & 15.5074 & 1.28675 & 0.00802938 & 12.182 & 1.15405 & 0.0253979 & 16.1415 \\
\hline & 3 & 1.10802 & 0.0488962 & 15.1773 & 1.13015 & 0.0230688 & 13.6827 & - & - & - \\
\hline \multirow{3}{*}{0.8} & 1 & 0.48547 & 0.0337892 & 15.1019 & 0.45375 & 0.0112144 & 12.8336 & 0.460708 & 0.0285934 & 17.2382 \\
\hline & 2 & 0.46327 & 0.0255848 & 15.3979 & 0.57618 & 0.00709028 & 12.1322 & 0.485456 & 0.0224305 & 15.9939 \\
\hline & 3 & 0.45522 & 0.0431833 & 14.995 & 0.46884 & 0.0203734 & 13.55 & - & - & - \\
\hline \multirow{3}{*}{0.9} & 1 & 0.02477 & 0.0298414 & 14.9874 & 0.00201 & 0.00990366 & 12.777 & 0.0074621 & 0.0252526 & 17.0938 \\
\hline & 2 & 0.0091 & 0.0225955 & 15.3123 & 0.08646 & 0.00626064 & 12.0933 & 0.0242636 & 0.0198097 & 15.8785 \\
\hline & 3 & 0.00432 & 0.0381379 & 14.8521 & 0.01272 & 0.017993 & 13.4463 & - & - & - \\
\hline
\end{tabular}

Table 7: The Optimal policy variable for Gamma distribution at $\beta=0.9$

\begin{tabular}{c|c|c|c|c|c|c}
\hline Item & source & $Q^{*}{ }_{m r s}$ & $\operatorname{Min} \mathrm{E}(\mathrm{TC})$ & $\tilde{Q}^{*}{ }_{\text {mrs }}$ & Source & $\operatorname{Min} \mathrm{E}(\widetilde{\mathrm{TC}})$ \\
\hline $\mathbf{1}$ & 3 & 0.0380405 & & 0.0381379 & 3 & \\
2 & 2 & 0.00714771 & 44.1944 & 0.00626064 & 2 & 42.823 \\
$\mathbf{3}$ & 2 & 0.0203673 & & 0.0198097 & 2 & \\
\hline
\end{tabular}

Table 8: The results of crisp and fuzzy values for Beta distribution

\begin{tabular}{cc|lll|lll|lcr}
\hline \multirow{2}{*}{$\beta_{r}$} & \multirow{2}{*}{$\backsim$} & \multicolumn{3}{|c|}{ Item 1 } & \multicolumn{3}{c|}{ Item 2 } & \multicolumn{3}{c}{ Item 3 } \\
\cline { 3 - 11 } & & $\lambda_{h r}$ & $Q_{m 1}$ & $E(T C)_{1}$ & $\lambda_{h r}$ & $Q_{m 2}$ & $E(T C)_{2}$ & $\lambda_{h r}$ & $Q_{m 3}$ & $E(T C)_{3}$ \\
\hline \multirow{3}{*}{0.1} & 1 & 18.8835 & 0.00645563 & 15.3247 & 32.7975 & 0.00211678 & 13.2814 & 20.3984 & 0.00497979 & 17.4085 \\
& 2 & 20.2663 & 0.00450279 & 15.6756 & 27.376 & 0.00147583 & 12.546 & 18.9877 & 0.00425484 & 16.2179 \\
& 3 & 18.7265 & 0.00815965 & 15.1627 & 23.3025 & 0.00384341 & 13.8613 & - & - & - \\
\hline \multirow{2}{*}{0.2} & 1 & 12.7003 & 0.00568945 & 15.2542 & 22.2677 & 0.00186729 & 13.2197 & 13.7389 & 0.00438996 & 17.3196 \\
& 2 & 13.6464 & 0.00396985 & 15.6221 & 18.5344 & 0.00130214 & 12.5096 & 12.7652 & 0.00375143 & 16.1464
\end{tabular}




\begin{tabular}{|c|c|c|c|c|c|c|c|c|c|c|}
\hline & 3 & 12.5981 & 0.0071892 & 15.0756 & 15.7353 & 0.00338898 & 13.7824 & - & - & - \\
\hline & 1 & 8.43809 & 0.0050149 & 15.1989 & 15.018 & 0.00164734 & 13.1716 & 9.15068 & 0.00387046 & 17.2499 \\
\hline \multirow[t]{3}{*}{0.3} & 2 & 9.0861 & 0.00350037 & 15.5802 & 12.4476 & 0.00114897 & 12.4812 & 8.47885 & 0.00330794 & 16.0904 \\
\hline & 3 & 8.37116 & 0.00633517 & 15.0072 & 10.5233 & 0.00298858 & 13.7207 & - & - & - \\
\hline & 1 & 5.50087 & 0.00442089 & 15.1557 & 10.027 & 0.00145339 & 13.134 & 5.99004 & 0.00341281 & 17.1954 \\
\hline \multirow[t]{3}{*}{0.4} & 2 & 5.94509 & 0.00308673 & 15.5474 & 8.2575 & 0.00101387 & 12.459 & 5.52673 & 0.00291717 & 16.0466 \\
\hline & 3 & 5.45678 & 0.00558341 & 14.9536 & 6.93406 & 0.00263574 & 13.6725 & - & - & - \\
\hline & 1 & 3.47717 & 0.0038977 & 15.1218 & 6.59116 & 0.00128235 & 13.1046 & 3.81312 & 0.00300958 & 17.1527 \\
\hline \multirow[t]{3}{*}{0.5} & 2 & 3.78191 & 0.00272224 & 15.5217 & 5.37298 & 0.000894706 & 12.4416 & 3.49373 & 0.00257281 & 16.0124 \\
\hline & 3 & 3.44795 & 0.00492153 & 14.9116 & 4.46246 & 0.00232477 & 13.6348 & - & - & - \\
\hline & 1 & 2.05572 & 0.0034681 & 15.0952 & 4.22579 & 0.00113152 & 13.0816 & 2.31394 & 0.00265425 & 17.1194 \\
\hline \multirow[t]{3}{*}{0.6} & 2 & 2.2923 & 0.00240101 & 15.5017 & 3.3873 & 0.000789585 & 12.4281 & 2.09384 & 0.00226929 & 15.9857 \\
\hline & 3 & 2.06365 & 0.00433866 & 14.8786 & 2.76067 & 0.00205065 & 13.6053 & - & - & - \\
\hline & 1 & 1.12295 & 0.00303073 & 15.0745 & 2.59749 & 0.000998478 & 13.0637 & 1.2816 & 0.00234108 & 17.0933 \\
\hline \multirow[t]{3}{*}{0.7} & 2 & 1.26663 & 0.00211786 & 15.486 & 2.02034 & 0.000696848 & 12.4175 & 1.12996 & 0.00200175 & 15.9647 \\
\hline & 3 & 1.10991 & 0.00382526 & 14.8528 & 1.58898 & 0.001809 & 13.5822 & - & - & - \\
\hline & 1 & 0.4617 & 0.00267289 & 15.0582 & 1.47657 & 0.000881127 & 13.0497 & 0.57079 & 0.00206503 & 17.0729 \\
\hline \multirow[t]{2}{*}{0.8} & 2 & 0.56045 & 0.00186824 & 15.4738 & 1.07932 & 0.000615029 & 12.4093 & 0.46634 & 0.00176588 & 15.9484 \\
\hline & 3 & 0.45293 & 0.00337299 & 14.8326 & 0.78232 & 0.00159593 & 13.5642 & - & - & - \\
\hline \multirow{3}{*}{0.9} & 1 & 0.00637 & 0.0023575 & 15.0455 & 0.70492 & 0.000777606 & 13.0388 & 0.08139 & 0.00182167 & 17.057 \\
\hline & 2 & 0.07425 & 0.00164817 & 15.4642 & 0.43151 & 0.000542838 & 12.4029 & 0.00945 & 0.00155792 & 15.9356 \\
\hline & 3 & 0.00043 & 0.0029745 & 14.8169 & 0.22697 & 0.00140806 & 13.5502 & - & - & - \\
\hline \multirow[t]{2}{*}{$\tilde{\beta}_{r}$} & & $\tilde{\lambda}_{h r}$ & $\tilde{Q}_{m 1}$ & $E(\widetilde{T C})_{1}$ & $\tilde{\lambda}_{h r}$ & $\tilde{Q}_{m 2}$ & $E(\widetilde{T C})_{2}$ & $\tilde{\lambda}_{h r}$ & $\tilde{Q}_{m 3}$ & $E(\widetilde{T C})_{3}$ \\
\hline & 1 & 19.0387 & 0.00640491 & 14.8916 & 18.8488 & 0.00204737 & 12.7323 & 18.8253 & 0.00536264 & 16.9747 \\
\hline \multirow[t]{3}{*}{0.1} & 2 & 19.0978 & 0.00472734 & 15.2422 & 18.8576 & 0.001393 & 12.063 & 18.8336 & 0.00425151 & 15.7847 \\
\hline & 3 & 19.8316 & 0.00776139 & 14.7304 & 19.6747 & 0.00365178 & 13.3627 & - & - & - \\
\hline & 1 & 12.807 & 0.00564481 & 14.8229 & 12.6646 & 0.0018061 & 12.6978 & 12.6566 & 0.00472712 & 16.8877 \\
\hline \multirow[t]{3}{*}{0.2} & 2 & 12.8423 & 0.00416763 & 15.1905 & 12.6701 & 0.0012291 & 12.0393 & 12.659 & 0.0037485 & 15.715 \\
\hline & 3 & 13.3583 & 0.00683875 & 14.6448 & 13.2366 & 0.00322014 & 13.2999 & - & - & - \\
\hline & 1 & 8.51153 & 0.00497559 & 14.769 & 8.40705 & 0.00159338 & 12.6709 & 8.40595 & 0.00416744 & 16.8196 \\
\hline \multirow[t]{3}{*}{0.3} & 2 & 8.53275 & 0.00367461 & 15.1501 & 8.41062 & 0.00108455 & 12.0208 & 8.40575 & 0.00330536 & 15.6604 \\
\hline & 3 & 8.8942 & 0.0060267 & 14.5776 & 8.80253 & 0.00283981 & 13.2507 & - & - & - \\
\hline & 1 & 5.5514 & 0.00438627 & 14.7268 & 5.47593 & 0.00140581 & 12.6499 & 5.47757 & 0.00367444 & 16.7663 \\
\hline \multirow[t]{3}{*}{0.4} & 2 & 5.56428 & 0.00324025 & 15.1184 & 5.4784 & 0.000957047 & 12.0063 & 5.47638 & 0.0029149 & 15.6177 \\
\hline & 3 & 5.81664 & 0.00531184 & 14.5249 & 5.74911 & 0.00250462 & 13.2123 & - & - & - \\
\hline & 1 & 3.51193 & 0.00386721 & 14.6937 & 3.45812 & 0.00124039 & 12.6335 & 3.46048 & 0.0032401 & 16.7245 \\
\hline \multirow[t]{3}{*}{0.5} & 2 & 3.51982 & 0.00285753 & 15.0936 & 3.45984 & 0.00084458 & 11.9951 & 3.45908 & 0.0025708 & 15.5844 \\
\hline & 3 & 3.69556 & 0.00468239 & 14.4837 & 3.64657 & 0.00220919 & 13.1823 & - & - & - \\
\hline & 1 & 2.10704 & 0.00340994 & 14.6679 & 2.06899 & 0.00109451 & 12.6206 & 2.07125 & 0.0028574 & 16.6919 \\
\hline \multirow[t]{3}{*}{0.6} & 2 & 2.11193 & 0.00252024 & 15.0743 & 2.07026 & 0.000745365 & 11.9863 & 2.06997 & 0.00226753 & 15.5583 \\
\hline & 3 & 2.23403 & 0.00412803 & 14.4513 & 2.19891 & 0.00194877 & 13.1588 & - & - & - \\
\hline & 1 & 1.13941 & 0.00300705 & 14.6476 & 1.11273 & 0.000965835 & 12.6106 & 1.11457 & 0.00252013 & 16.6664 \\
\hline \multirow[t]{3}{*}{0.7} & 2 & 1.14248 & 0.00222296 & 15.0591 & 1.11367 & 0.000657834 & 11.9794 & 1.11353 & 0.00200019 & 15.5379 \\
\hline & 3 & 1.22715 & 0.00363973 & 14.426 & 1.20223 & 0.00171917 & 13.1405 & - & - & - \\
\hline & 1 & 0.47033 & 0.00265202 & 14.6318 & 0.45444 & 0.000852332 & 12.6028 & 0.45583 & 0.00222286 & 16.6464 \\
\hline \multirow[t]{3}{*}{0.8} & 2 & 0.47499 & 0.0019609 & 15.0473 & 0.45514 & 0.000580607 & 11.974 & 0.45502 & 0.00176451 & 15.5219 \\
\hline & 3 & 0.53361 & 0.00320952 & 14.4062 & 0.51605 & 0.00151673 & 13.1261 & - & - & - \\
\hline & 1 & 0.04142 & 0.00233912 & 14.6194 & 0.00127 & 0.000752203 & 12.5967 & 0.002265 & 0.00196081 & 16.6308 \\
\hline \multirow[t]{2}{*}{0.9} & 2 & 0.01543 & 0.00172987 & 15.0381 & 0.0018 & 0.000512466 & 11.9699 & 0.00167 & 0.00155671 & 15.5095 \\
\hline & 3 & 0.05596 & 0.00283045 & 14.3907 & 0.04367 & 0.00133821 & 13.1149 & - & - & - \\
\hline
\end{tabular}

Table 9: The results of crisp and fuzzy values for Beta distribution

\begin{tabular}{ccccccc}
\hline Item & source & $\boldsymbol{Q}^{*}{ }_{\text {mrs }}$ & Min E(TC) & $\widetilde{\boldsymbol{Q}}^{*}{ }_{\text {mrs }}$ & Source & $\operatorname{Min} \mathbf{E}(\widetilde{\mathbf{T C}})$ \\
\hline $\mathbf{1}$ & 3 & 0.0029745 & & 0.00283045 & 3 & \\
$\mathbf{2}$ & 2 & 0.000542838 & 43.1554 & 0.000512466 & 2 & 41.8701 \\
$\mathbf{3}$ & 2 & 0.00155792 & & 0.00155671 & 2 & \\
\hline
\end{tabular}


Table 10: The results of crisp and fuzzy values for Raylieph distribution

\begin{tabular}{|c|c|c|c|c|c|c|c|c|c|c|}
\hline \multirow{2}{*}{$\beta_{r}$} & \multirow{2}{*}{$\begin{array}{l}\text { O } \\
\stackrel{\complement}{c} \\
\tilde{D}\end{array}$} & \multicolumn{3}{|c|}{ Item 1} & \multicolumn{3}{|c|}{ Item 2} & \multicolumn{3}{|c|}{ Item 3} \\
\hline & & $\lambda_{h r}$ & $Q_{m 1}$ & $E(T C)_{1}$ & $\lambda_{h r}$ & $Q_{m 2}$ & $E(T C)_{2}$ & $\lambda_{h r}$ & $Q_{m 3}$ & $E(T C)_{3}$ \\
\hline \multirow{3}{*}{0.1} & 1 & 18.4856 & 0.0487883 & 16.8822 & 18.6994 & 0.0163127 & 13.9652 & 18.586 & 0.0405919 & 19.3678 \\
\hline & 2 & 20.1581 & 0.0339157 & 16.858 & 18.7981 & 0.011386 & 13.0583 & 18.6972 & 0.0324043 & 17.7964 \\
\hline & 3 & 18.43 & 0.0608416 & 17.0907 & 18.5892 & 0.029363 & 15.2198 & - & - & - \\
\hline \multirow{3}{*}{0.2} & 1 & 12.4609 & 0.0430716 & 16.4752 & 12.5827 & 0.014405 & 13.7541 & 12.5237 & 0.0358379 & 18.8543 \\
\hline & 2 & 13.6034 & 0.0299452 & 16.5476 & 12.6469 & 0.0100548 & 12.9096 & 12.5939 & 0.0286111 & 17.3817 \\
\hline & 3 & 12.4321 & 0.0537074 & 16.589 & 12.5171 & 0.0259263 & 14.8453 & - & - & - \\
\hline \multirow{3}{*}{0.3} & 1 & 8.29582 & 0.0380264 & 16.1557 & 8.36421 & 0.0127205 & 13.589 & 8.33523 & 0.0316418 & 18.4516 \\
\hline & 2 & 9.07707 & 0.0264403 & 16.3043 & 8.40605 & 0.00887934 & 12.7935 & 8.37972 & 0.0252626 & 17.0569 \\
\hline & 3 & 8.28179 & 0.0474124 & 16.1944 & 8.32519 & 0.0228926 & 14.5521 & - & - & - \\
\hline \multirow{3}{*}{0.4} & 1 & 5.41761 & 0.0335735 & 15.9051 & 5.45524 & 0.0112332 & 13.46 & 5.44238 & 0.027938 & 18.136 \\
\hline & 2 & 5.95222 & 0.0233463 & 16.1139 & 5.48266 & 0.00784136 & 12.7028 & 5.47067 & 0.0223066 & 16.8026 \\
\hline & 3 & 5.41147 & 0.0418573 & 15.8844 & 5.43219 & 0.0202142 & 14.3226 & - & - & - \\
\hline \multirow{3}{*}{0.5} & 1 & 3.42939 & 0.0296431 & 15.7086 & 3.44957 & 0.00991985 & 13.3593 & 3.44503 & 0.0246684 & 17.8888 \\
\hline & 2 & 3.79551 & 0.0206148 & 15.9648 & 3.46758 & 0.00692478 & 12.632 & 3.46308 & 0.0196969 & 16.6036 \\
\hline & 3 & 3.42732 & 0.0369547 & 15.6411 & 3.43597 & 0.0178497 & 14.1431 & - & - & - \\
\hline \multirow{3}{*}{0.6} & 1 & 2.05644 & 0.0261736 & 15.5546 & 2.06683 & 0.00876017 & 13.2805 & 2.06634 & 0.021782 & 17.6953 \\
\hline & 2 & 2.30733 & 0.0182033 & 15.8481 & 2.07871 & 0.00611538 & 12.5767 & 2.07789 & 0.0173929 & 16.4479 \\
\hline & 3 & 2.05632 & 0.0326276 & 15.4503 & 2.05887 & 0.015762 & 14.0026 & - & - & - \\
\hline \multirow{3}{*}{0.7} & 1 & 1.10862 & 0.0231109 & 15.4341 & 1.11365 & 0.00773613 & 13.2191 & 1.11491 & 0.0192338 & 17.5439 \\
\hline & 2 & 1.28065 & 0.0160742 & 15.7569 & 1.12151 & 0.00540062 & 12.5336 & 1.12233 & 0.0153587 & 16.3261 \\
\hline & 3 & $1.1-933$ & 0.0288082 & 15.3007 & 1.10902 & 0.0139187 & 13.8928 & - & - & - \\
\hline \multirow{3}{*}{0.8} & 1 & 0.45446 & 0.020407 & 15.3397 & 0.45663 & 0.00683185 & 13.1711 & 0.458474 & 0.016984 & 17.4254 \\
\hline & 2 & 0.57247 & 0.0141944 & 15.6855 & 0.46185 & 0.00476943 & 12.4999 & 0.463264 & 0.0135626 & 16.2309 \\
\hline & 3 & 0.45544 & 0.0254366 & 15.1835 & 0.454 & 0.0122911 & 13.807 & - & - & - \\
\hline \multirow{3}{*}{0.9} & 1 & 0.00309 & 0.0180198 & 15.2659 & 0.00379 & 0.00603332 & 13.1336 & 0.00565 & 0.0149977 & 17.3328 \\
\hline & 2 & 0.08408 & 0.0125346 & 15.6298 & 0.00727 & 0.00421203 & 12.4736 & 0.00876 & 0.0119767 & 16.1565 \\
\hline & 3 & 0.00406 & 0.0224602 & 15.0918 & 0.00227 & 0.010854 & 13.74 & - & - & - \\
\hline \multirow[t]{2}{*}{$\tilde{\beta}_{r}$} & & $\tilde{\lambda}_{h r}$ & $\tilde{Q}_{m 1}$ & $E(\widetilde{T C})_{1}$ & $\tilde{\lambda}_{h r}$ & $\tilde{Q}_{m 2}$ & $E(\widetilde{T C})_{2}$ & $\tilde{\lambda}_{h r}$ & $\tilde{Q}_{m 3}$ & $E(\widetilde{T C})_{3}$ \\
\hline & 1 & 18.7746 & 0.0480898 & 16.4111 & 18.6608 & 0.0157942 & 13.4923 & 18.6338 & 0.0403598 & 18.8959 \\
\hline 0.1 & 2 & 18.5042 & 0.0364375 & 16.3828 & 18.7169 & 0.0107989 & 12.5851 & 18.5623 & 0.0323519 & 17.3238 \\
\hline & 3 & 18.447 & 0.0610933 & 16.6186 & 18.5913 & 0.0290323 & 14.7475 & - & - & - \\
\hline & 1 & 12.66 & 0.0424551 & 16.014 & 12.5557 & 0.0139471 & 13.2916 & 12.5566 & 0.035633 & 18.3925 \\
\hline 0.2 & 2 & 12.464 & 0.0321711 & 16.0836 & 12.5904 & 0.00953643 & 12.447 & 12.5008 & 0.0285648 & 16.9195 \\
\hline & 3 & 12.4441 & 0.0539295 & 16.1271 & 12.5182 & 0.0256344 & 14.3833 & - & - & - \\
\hline & 1 & 6.43297 & 0.0374823 & 15.7023 & 8.3453 & 0.0123163 & 13.1347 & 8.35778 & 0.031461 & 17.9978 \\
\hline 0.3 & 2 & 8.29197 & 0.0284052 & 15.849 & 8.3684 & 0.0084216 & 12.3391 & 8.31548 & 0.0252217 & 16.6028 \\
\hline & 3 & 8.29019 & 0.0476084 & 15.7405 & 8.32582 & 0.0226349 & 14.0981 & - & - & - \\
\hline & 1 & 5.51208 & 0.0330933 & 15.4577 & 5.4421 & 0.0108762 & 13.0122 & 5.45788 & 0.0277784 & 17.6884 \\
\hline 0.4 & 2 & 5.41134 & 0.0250808 & 15.6653 & 5.45546 & 0.00743715 & 12.2549 & 5.42634 & 0.0222705 & 16.3548 \\
\hline & 3 & 5.41734 & 0.0420303 & 15.4368 & 5.43253 & 0.0199867 & 13.8749 & - & - & - \\
\hline & 1 & 3.49447 & 0.0292192 & 15.266 & 3.44041 & 0.00960466 & 12.9164 & 3.45568 & 0.0245275 & 17.4461 \\
\hline 0.5 & 2 & 3.42286 & 0.0221461 & 15.5214 & 3.44873 & 0.00656784 & 12.1891 & 3.43248 & 0.0196651 & 16.1608 \\
\hline & 3 & 3.43142 & 0.0371074 & 15.1984 & 3.43615 & 0.0176488 & 13.7003 & - & - & - \\
\hline & 1 & 2.10127 & 0.0257994 & 15.1159 & 2.06046 & 0.00848184 & 12.8416 & 2.07366 & 0.0216576 & 17.2564 \\
\hline 0.6 & 2 & 2.05058 & 0.0195553 & 15.4089 & 2.06566 & 0.00580018 & 12.1377 & 2.05679 & 0.0173648 & 16.0089 \\
\hline & 3 & 2.05918 & 0.0327623 & 15.0114 & 2.05896 & 0.0155846 & 13.5637 & - & - & - \\
\hline & 1 & 1.1395 & 0.0227805 & 14.9983 & 1.10922 & 0.00749035 & 12.7831 & 1.11994 & 0.019124 & 17.108 \\
\hline 0.7 & 2 & 1.10378 & 0.0172678 & 15.3208 & 1.11248 & 0.00512227 & 12.0976 & 1.10777 & 0.0153339 & 15.8902 \\
\hline & 3 & 1.11132 & 0.0289271 & 14.8648 & 1.10905 & 0.0137621 & 13.457 & - & - & - \\
\hline & 1 & 0.47574 & 0.0201153 & 14.9062 & 0.45356 & 0.00661482 & 12.7375 & 0.461931 & 0.0168871 & 16.9919 \\
\hline 0.8 & 2 & 0.45063 & 0.0152483 & 15.252 & 0.4556 & 0.00452362 & 12.0663 & 0.453224 & 0.0135407 & 15.7974 \\
\hline & 3 & 0.45683 & 0.0255416 & 14.75 & 0.45397 & 0.0121529 & 13.3735 & - & - & - \\
\hline & 1 & 0.01775 & 0.0177623 & 14.8342 & 0.00166 & 0.00584166 & 12.7019 & 0.00803 & 0.0149121 & 16.9011 \\
\hline 0.9 & 2 & 0.00014 & 0.0134651 & 15.1981 & 0.00295 & 0.00399495 & 12.0419 & 0.00183 & 0.0119574 & 15.7249 \\
\hline & 3 & 0.00502 & 0.0225529 & 14.6601 & 0.00227 & 0.0107319 & 13.3083 & - & - & - \\
\hline
\end{tabular}


Table 11: The Optimal policy variable for Raylieph distribution at $\beta=0.9$

\begin{tabular}{ccccccc}
\hline Item & source & $Q^{*}{ }_{m r s}$ & Min E(TC) & $\tilde{Q}^{*}{ }_{m r s}$ & Source & Min E( $\widetilde{\mathrm{TC}})$ \\
\hline 1 & 3 & 0.0224602 & & 0.0225529 & 3 & \\
2 & 2 & 0.00421203 & 43.7219 & 0.00399495 & 2 & 42.4269 \\
3 & 2 & 0.0119767 & & 0.0119574 & 2 & \\
\hline
\end{tabular}

Table 12: The results of crisp and fuzzy values for Erlang distribution

\begin{tabular}{|c|c|c|c|c|c|c|c|c|c|c|}
\hline \multirow{2}{*}{$\beta_{r}$} & \multirow[t]{2}{*}{$n$} & \multicolumn{3}{|c|}{ Item 1} & \multicolumn{3}{|c|}{ Item 2} & \multicolumn{3}{|c|}{ Item 3} \\
\hline & & $\lambda_{h r}$ & $Q_{m 1}$ & $E(T C)_{1}$ & $\lambda_{h r}$ & $Q_{m 2}$ & $E(T C)_{2}$ & $\lambda_{h r}$ & $Q_{m 3}$ & $E(T C)_{3}$ \\
\hline \multirow{3}{*}{0.1} & 1 & 18.8942 & 0.0446332 & 16.8831 & 18.7561 & 0.0257575 & 14.6244 & 18.5436 & 0.0380102 & 19.3661 \\
\hline & 2 & 18.5063 & 0.0321026 & 16.7612 & 18.6905 & 0.0152993 & 13.3434 & 18.5023 & 0.0306076 & 17.7939 \\
\hline & 3 & 18.4067 & 0.056787 & 17.0916 & 18.4789 & 0.0336475 & 15.5893 & - & - & - \\
\hline \multirow{4}{*}{0.2} & 1 & 12.7482 & 0.0394131 & 16.4753 & 12.6346 & 0.0227467 & 14.2703 & 12.4997 & 0.0335656 & 18.8525 \\
\hline & 2 & 12.4683 & 0.0283495 & 16.4716 & 12.5792 & 0.0135114 & 13.1326 & 12.4641 & 0.0270294 & 17.3795 \\
\hline & 3 & 12.4228 & 0.0501425 & 16.589 & 12.4508 & 0.0297136 & 15.1351 & - & - & - \\
\hline & 1 & 8.49751 & 0.0348043 & 16.1553 & 8.40787 & 0.020088 & 13.9931 & 8.32196 & 0.0296413 & 18.4499 \\
\hline \multirow[t]{3}{*}{0.3} & 2 & 8.29683 & 0.0250355 & 16.2448 & 8.36334 & 0.0119325 & 12.9678 & 8.29304 & 0.0238699 & 17.055 \\
\hline & 3 & 8.27971 & 0.0442769 & 16.1939 & 8.28569 & 0.02624 & 14.7792 & - & - & - \\
\hline & 1 & 5.55889 & 0.030735 & 15.9045 & 5.49015 & 0.0177403 & 13.7762 & 5.4353 & 0.0261762 & 18.1344 \\
\hline \multirow[t]{3}{*}{0.4} & 2 & 5.41583 & 0.0221092 & 16.0672 & 5.45567 & 0.0105381 & 12.839 & 5.4127 & 0.0210799 & 16.801 \\
\hline & 3 & 5.41277 & 0.0390985 & 15.8837 & 5.4087 & 0.0231728 & 14.5005 & - & - & - \\
\hline & 1 & 3.52821 & 0.0271419 & 15.7079 & 3.47659 & 0.015667 & 13.6066 & 3.44144 & 0.0231164 & 17.8874 \\
\hline \multirow[t]{3}{*}{0.5} & 2 & 3.42671 & 0.0195251 & 15.9282 & 3.45004 & 0.0093076 & 12.7385 & 3.42424 & 0.0186161 & 16.6022 \\
\hline & 3 & 3.42995 & 0.0345265 & 15.6402 & 3.42213 & 0.0204643 & 14.2824 & - & - & - \\
\hline & 1 & 2.12543 & 0.0239692 & 15.5539 & 2.08727 & 0.0138361 & 13.474 & 2.06467 & 0.0204145 & 17.6941 \\
\hline \multirow[t]{3}{*}{0.6} & 2 & 2.05373 & 0.0172431 & 15.8195 & 2.06781 & 0.0082193 & 12.6599 & 2.0518 & 0.0164405 & 16.4467 \\
\hline & 3 & 2.05921 & 0.0304898 & 15.4494 & 2.05077 & 0.0180725 & 14.1117 & - & - & - \\
\hline & 1 & 1.15674 & 0.0211675 & 15.4334 & 1.12883 & 0.0122193 & 13.3704 & 1.11426 & 0.0180286 & 17.5428 \\
\hline \multirow[t]{3}{*}{0.7} & 2 & 1.10623 & 0.015228 & 15.7345 & 1.11455 & 0.0072589 & 12.5986 & 1.10478 & 0.0145192 & 16.3252 \\
\hline & 3 & 1.11202 & 0.0269254 & 15.2999 & 1.10432 & 0.0159604 & 13.9783 & - & - & - \\
\hline & 1 & 0.48798 & 0.0186935 & 15.3391 & 0.46778 & 0.0107914 & 13.2894 & 0.458843 & 0.0159216 & 17.4245 \\
\hline \multirow[t]{3}{*}{0.8} & 2 & 0.45251 & 0.0134484 & 15.668 & 0.4574 & 0.00641072 & 12.5506 & 0.451431 & 0.0128225 & 16.2302 \\
\hline & 3 & 0.45773 & 0.023778 & 15.1827 & 0.45126 & 0.0140953 & 13.8739 & - & - & - \\
\hline & 1 & 0.0264 & 0.0165088 & 15.2653 & 0.01189 & 0.00953041 & 13.2262 & 0.00576 & 0.014061 & 17.332 \\
\hline \multirow[t]{2}{*}{0.9} & 2 & 0.00155 & 0.0118769 & 15.6161 & 0.00441 & 0.00566166 & 12.5132 & 0.00077 & 0.0113241 & 16.1559 \\
\hline & 3 & 0.0059 & 0.0209987 & 15.0911 & 0.00074 & 0.0124481 & 13.7923 & - & - & - \\
\hline \multirow[t]{2}{*}{$\tilde{\beta}_{r}$} & & $\tilde{\lambda}_{h r}$ & $\tilde{Q}_{m 1}$ & $E(\widetilde{T C})_{1}$ & $\tilde{\lambda}_{h r}$ & $\tilde{Q}_{m 2}$ & $E(\widetilde{T C})_{2}$ & $\tilde{\lambda}_{h r}$ & $\tilde{Q}_{m 3}$ & $E(\widetilde{T C})_{3}$ \\
\hline & 1 & 18.4037 & 0.0457318 & 16.4095 & 18.5695 & 0.0256674 & 14.1518 & 18.4728 & 0.0380189 & 18.8938 \\
\hline \multirow[t]{3}{*}{0.1} & 2 & 19.9463 & 0.0317638 & 16.384 & 18.6373 & 0.0148251 & 12.8706 & 18.5062 & 0.0303469 & 17.3217 \\
\hline & 3 & 18.436 & 0.0569856 & 16.6196 & 18.5403 & 0.0333411 & 15.1173 & - & - & - \\
\hline & 1 & 12.4103 & 0.040383 & 16.0124 & 12.5058 & 0.0226671 & 13.808 & 12.4508 & 0.0335733 & 18.3905 \\
\hline \multirow[t]{3}{*}{0.2} & 2 & 13.4621 & 0.0280504 & 16.084 & 12.542 & 0.0130926 & 12.6702 & 12.4665 & 0.0267993 & 16.9175 \\
\hline & 3 & 12.4433 & 0.0503178 & 16.1271 & 12.493 & 0.029443 & 14.6733 & - & - & - \\
\hline & 1 & 8.26471 & 0.0356606 & 15.7008 & 8.31889 & 0.0200178 & 13.5389 & 8.28819 & 0.0296481 & 17.9959 \\
\hline \multirow[t]{3}{*}{0.3} & 2 & 8.98264 & 0.0247714 & 15.849 & 8.3374 & 0.0115627 & 12.5136 & 8.29459 & 0.0236666 & 16.6011 \\
\hline & 3 & 8.29395 & 0.0444317 & 15.74 & 8.31459 & 0.0260011 & 14.3254 & - & - & - \\
\hline & 1 & 5.39851 & 0.0314911 & 15.4564 & 5.42874 & 0.0176783 & 13.3284 & 5.41199 & 0.0261822 & 17.6867 \\
\hline 0.4 & 2 & 5.88902 & 0.0218759 & 15.665 & 5.43762 & 0.0102115 & 12.3912 & 5.41366 & 0.0209004 & 16.3533 \\
\hline & 3 & 5.4227 & 0.0392351 & 15.4361 & 5.42855 & 0.0229618 & 14.0529 & - & - & - \\
\hline & 1 & 3.41772 & 0.0278095 & 15.2649 & 3.43423 & 0.0156122 & 13.1638 & 3.42536 & 0.0231217 & 17.4447 \\
\hline 0.5 & 2 & 3.75311 & 0.0193191 & 15.521 & 3.4379 & 0.00901832 & 12.2956 & 3.42484 & 0.0184576 & 16.1595 \\
\hline & 3 & 3.43684 & 0.0346472 & 15.1976 & 3.43576 & 0.020278 & 13.8397 & - & - & - \\
\hline & 1 & 2.04932 & 0.0245587 & 15.1149 & 2.05802 & 0.0137878 & 13.0351 & 2.05357 & 0.0204192 & 17.2552 \\
\hline 0.6 & 2 & 2.27884 & 0.0170612 & 15.4084 & 2.0591 & 0.00796455 & 12.221 & 2.05219 & 0.0163005 & 16.0079 \\
\hline & 3 & 2.06401 & 0.0305962 & 15.0106 & 2.06013 & 0.017908 & 13.6729 & - & - & - \\
\hline & 1 & 1.1043 & 0.0216881 & 14.9974 & 1.10866 & 0.0121766 & 12.9345 & 1.10661 & 0.0180327 & 17.1069 \\
\hline 0.7 & 2 & 1.26146 & 0.0150674 & 15.3203 & 1.10851 & 0.00703392 & 12.1627 & 1.10502 & 0.0143956 & 15.8893 \\
\hline & 3 & 1.11535 & 0.0270194 & 14.864 & 1.11074 & 0.0158152 & 13.5424 & - & - & - \\
\hline 0.8 & 1 & 0.45185 & 0.0191533 & 14.9055 & 0.45386 & 0.0107537 & 12.8559 & 0.453059 & 0.0159253 & 16.991 \\
\hline
\end{tabular}




\begin{tabular}{cc|ccc|ccc|ccc} 
& 2 & 0.55954 & 0.0133066 & 15.2515 & 0.45321 & 0.00621204 & 12.1171 & 0.451591 & 0.0127133 & 15.7967 \\
& 3 & 0.46003 & 0.023861 & 14.7493 & 0.45568 & 0.013967 & 13.4404 & - & - \\
\hline \multirow{2}{*}{$\mathbf{0 . 9}$} & $\mathbf{1}$ & $\mathbf{0 . 0 0 1 5 2}$ & $\mathbf{0 . 0 1 6 9 1 4 8}$ & $\mathbf{1 4 . 8 3 3 7}$ & $\mathbf{0 . 0 0 2 3}$ & $\mathbf{0 . 0 0 9 4 9 7 0 9}$ & $\mathbf{1 2 . 7 9 4 5}$ & $\mathbf{0 . 0 0 2 1 2}$ & $\mathbf{0 . 0 1 4 0 6 4 2}$ & $\mathbf{1 6 . 9 0 0 4}$ \\
& $\mathbf{2}$ & $\mathbf{0 . 0 7 5 3 5}$ & $\mathbf{0 . 0 1 1 7 5 1 6}$ & $\mathbf{1 5 . 1 9 7 7}$ & $\mathbf{0 . 0 0 1 5}$ & $\mathbf{0 . 0 0 5 4 8 6 2 1}$ & $\mathbf{1 2 . 0 8 1 6}$ & $\mathbf{0 . 0 0 0 8 7}$ & $\mathbf{0 . 0 1 1 2 2 7 7}$ & $\mathbf{1 5 . 7 2 4 3}$ \\
& $\mathbf{3}$ & $\mathbf{0 . 0 0 7 5}$ & $\mathbf{0 . 0 2 1 0 7 2}$ & $\mathbf{1 4 . 6 5 9 4}$ & $\mathbf{0 . 0 0 3 8}$ & $\mathbf{0 . 0 1 2 3 3 4 8}$ & $\mathbf{1 3 . 3 6 0 7}$ & - & - & - \\
\hline
\end{tabular}

Table 13: The Optimal policy variable for Erlang distribution at $\beta=0.9$

\begin{tabular}{ccccccc}
\hline Item & Source & $\boldsymbol{Q}^{*}{ }_{\text {mrs }}$ & Min E(TC) & $\widetilde{\boldsymbol{Q}}^{*}{ }_{\text {mrs }}$ & Source & $\operatorname{Min} \mathbf{E}(\widetilde{\mathbf{T C}})$ \\
\hline $\mathbf{1}$ & 3 & 0.0209987 & & 0.021072 & 3 & \\
$\mathbf{2}$ & 2 & 0.00566166 & 43.7602 & 0.00548621 & 2 & 42.4653 \\
$\mathbf{3}$ & 2 & 0.0113241 & & 0.0112277 & 2 & \\
\hline
\end{tabular}

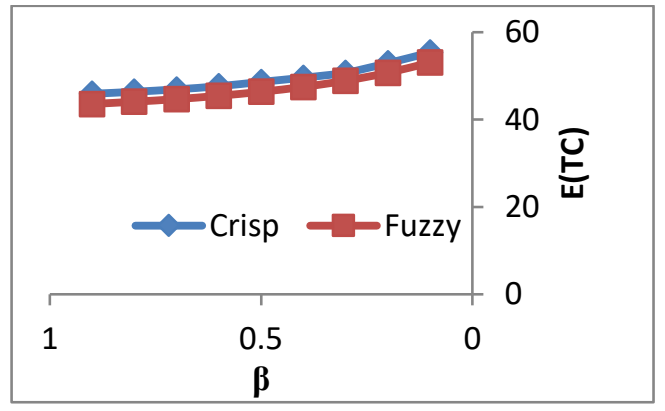

Figure 4: Crisp and fuzzy value for two-parameter exponential distribution

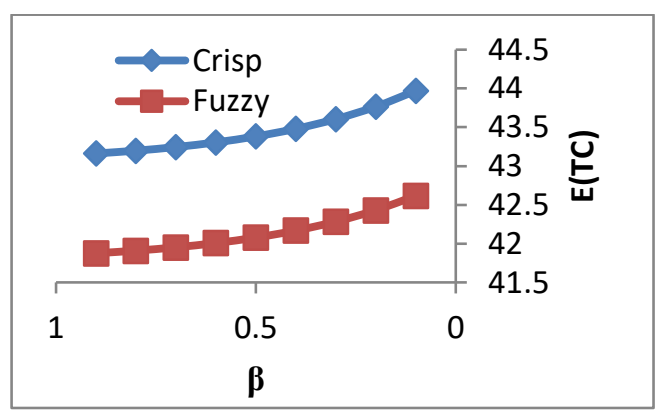

Figure 5: Crisp and fuzzy value for

Kumaraswamy distribution

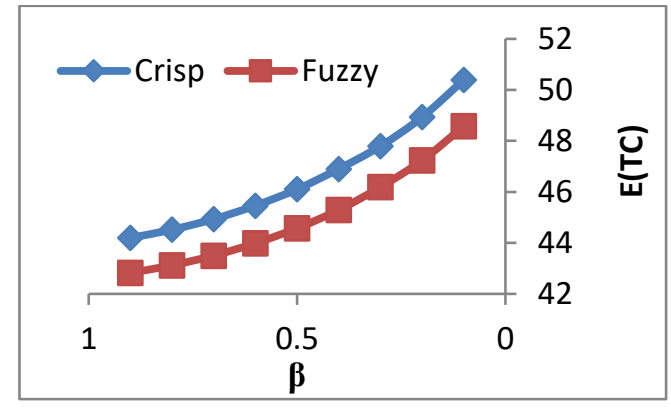

Figure 6: Crisp and fuzzy value for

Gamma distribution

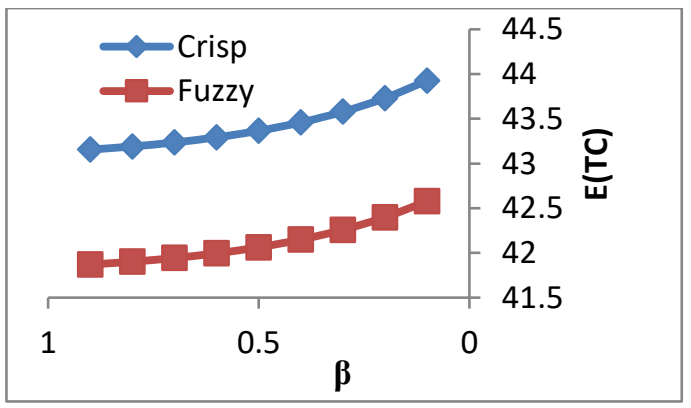

Figure 7: Crisp and fuzzy value for

Beta distribution 


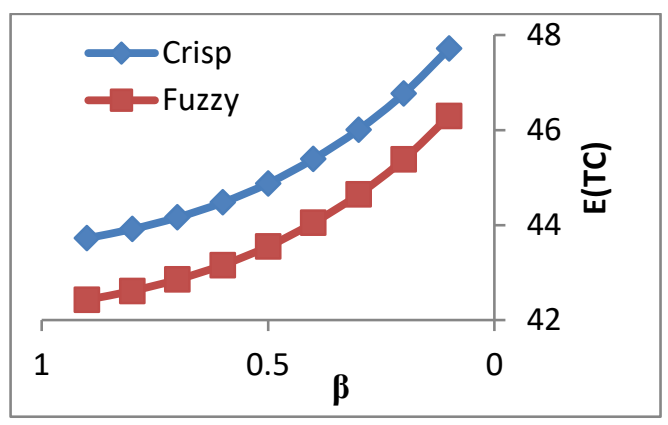

Figure 8: Crisp and fuzzy value for

Raylieph distribution

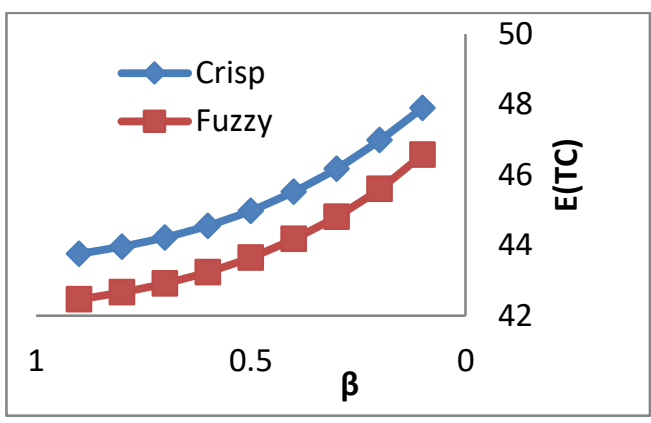

Figure 9: Crisp and fuzzy value for

Erlang distribution

\section{Conclusion}

This paper concerns with a multi-item multi-source (MIMS) constrained probabilistic periodic review inventory model. We determine the optimal maximum inventory level for a given $N$ that minimized the expected annual total cost under varying holding cost constraint using Lagrange multiplier technique for crisp and TFN environment. And we conclude that: the fuzzy environment is more closed to the practical situation than crisp number. Also, When $\beta$ convergence to 1 , the solution approaching to the optimal solution. Furthermore, under our assumption and from selected distributions: Raylieph distribution give the optimum expected annual total cost.

\section{References}

1. Abuo - El-Ata M., Fergany H. and El-Wakeel M., Probabilistic Multi-Item Inventory Model with Varying Order Cost under Two Restrictions: A Geometric Programming Approach, International Journal of Production Economics, 83 (2003) PP. 223-231.

2. Chiang C., A periodic review replenishment model with a refined delivery scenario, International Journal of Production Economics, 118 (2009) PP. 253-259

3. Chiang C., Optimal ordering policies for periodic-review systems with a refined intra-cycle time scale, Production, Manufacturing and Logistics, European Journal of Operational Research 177 (2007) PP. 872 881.

4. Chiang C., Optimal replenishment for a periodic review inventory system with two supply modes, European Journal of Operational Research 149 (2003) PP. 229-244

5. Chiang C., Periodic Review Inventory Models with Stochastic Supplier's Visit Intervals, International Journal of Production Economics, 115 (2008) PP. 433-438.

6. Chu K., Lung Y., Shing K. and Thomas N., Note on inventory model with a mixture of back orders and lost sales Peter, European Journal of Operational Research 159 (2004) PP. 470-475.

7. Fabrycky W. J. and Banks Jerry. (1967). Procurement and Inventory Systems: THEORY AND ANALYSIS. Newwork, Amsterdam, London.

8. Fergany H., Probabilistic Multi-Item Inventory Model with Varying Mixture Shortage Cost Under Restrictions, Springer Plus Journal of Springer Plus 5(1), (2016), PP. 1-13. 


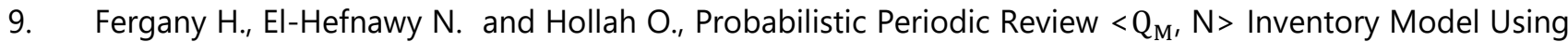
Lagrange Technique And Fuzzy Adaptive Particle Swarm Optimization, Journal of Mathematics and Statistics 10 (3) (2014) PP. 368-383.

10. Fergany H. and El-Wakeel M., Probabilistic Single - Item Inventory Problem with Varying Order Cost Under Two Linear Constraints. Journal of the Egyptian Mathematical Society, 12(1), (2004) PP. 71-81.

11. Konstantaras I. and Papachristos S., Production, Manufacturing and Logistics Optimal policy and holding cost stability regions in a periodic review inventory system with manufacturing and remanufacturing options, European Journal of Operational Research 178 (2007) PP. 433-448.

12. Rong M., Mahapatra N. and Maiti M., A multi-objective wholesaler-retailers inventory-distribution model with controllable lead-time based on probabilistic fuzzy set and triangular fuzzy number, Applied Mathematical Modelling 32 (2008) PP. 2670-2685.

13. Sadjadi S., Ghazanfari M. and Yousefli A., Fuzzy Pricing and Marketing Planning Model: A Possibilistic Geometric Programming Approach, Expert System Applied 37 (2010) PP. 3392-3397.

14. Silver A. and Robb J., Some insights regarding the optimal reorder period in periodic review inventory systems, International Journal of Production Economics 112 (2008) PP. 354-366

15. Yuyue S. and Hoong C., A periodic-review inventory model with application to the continuous-review obsolescence problem, European Journal of Operational Research 159 (2004) PP.110-120. 